\title{
Tetrahydrobiopterin activates brown adipose tissue and regulates systemic energy metabolism
}

\author{
Yasuo Oguri, ${ }^{1,2}$ Yoshihito Fujita, ${ }^{1}$ Abulizi Abudukadier, ${ }^{1}$ Akiko Ohashi, ${ }^{3}$ Tsuyoshi Goto, ${ }^{4}$ \\ Futoshi Furuya, ${ }^{1}$ Akio Obara, ${ }^{1}$ Toru Fukushima, ${ }^{1}$ Naomi Matsuo, ${ }^{1}$ Minji Kim, ${ }^{4}$ Masaya Hosokawa, ${ }^{1,5}$ \\ Teruo Kawada, ${ }^{4}$ Hiroyuki Hasegawa, ${ }^{3}$ and Nobuya Inagaki ${ }^{1}$ \\ 'Department of Diabetes, Endocrinology and Nutrition, Graduate School of Medicine, Kyoto University, Kyoto, Japan. \\ 2Japan Society for the Promotion of Science, Tokyo, Japan. ${ }^{3}$ Department of Anatomy, Nihon University School of Dentistry, \\ Tokyo, Japan. ${ }^{4}$ Laboratory of Molecular Function of Food, Division of Food Science and Biotechnology, Graduate School of \\ Agriculture, Kyoto University, Kyoto, Japan. ${ }^{5}$ Faculty of Human Sciences, Tezukayama Gakuin University, Osaka, Japan.
}

Brown adipose tissue (BAT) is a central organ that acts to increase energy expenditure; its regulatory factors could be clinically useful in the treatment of obesity. Tetrahydrobiopterin (BH4) is an essential cofactor of tyrosine hydroxylase and nitric oxide synthase (NOS). Although BH4 regulates the known regulatory factors of BAT, such as noradrenaline (NA) and NO, participation of BH4 in BAT function remains unclear. In the present study, we investigate the role of BH4 in the regulation of BAT. Hph-1 mice, a mouse model of BH4 deficiency, exhibit obesity, adiposity, glucose intolerance, insulin resistance, and impaired BAT function. Impaired BAT function was ameliorated together with systemic metabolic disturbances by BAT transplantation from BH4-sufficient mice (control mice) into BH4-deficient mice, strongly suggesting that BH4-induced BAT has a critical role in the regulation of systemic energy metabolism. Both NA derived from the sympathetic nerve and NO derived from endothelial NOS in the blood vessels participate in the regulation of BH4. In addition, a direct effect of BH4 in the stimulation of brown adipocytes via NO is implicated. Taken together, BH4 activates BAT and regulates systemic energy metabolism; this suggests an approach for metabolic disorders, such as obesity and diabetes.

Conflict of interest: The authors have declared that no conflict of interest exists.

Submitted: November 30, 2016 Accepted: March 29, 2017 Published: May 4, 2017

Reference information: JCI Insight. 2017;2(9):e91981. https:// doi.org/10.1172/jci.insight.91981.

\section{Introduction}

The prevalence of obesity is increasing all over the world, resulting in widespread insulin resistance and progression of type 2 diabetes $(1,2)$. Body weight control is a critical requirement in the treatment of type 2 diabetes. Obesity is induced by an imbalance of energy intake and energy expenditure; however, several approaches to correct the imbalance have been employed (3-5). Brown adipose tissue (BAT) acts to increase energy expenditure; it contains numerous mitochondria and has the remarkable capacity to induce thermogenesis by mitochondrial respiration thorough uncoupling protein 1 (UCP1). Recent studies reported that BAT is functionalized in adult humans and that BMI is inversely correlated with the amount of BAT, especially in older patients (6-9). Herein, BAT is focused on a therapeutic target for treatment of obesity and its associated metabolic disorders, such as type 2 diabetes (10-13). There are currently no approaches in clinical use; novel therapeutic approaches that effectively activate BAT are required.

Tetrahydrobiopterin (BH4) is an essential cofactor of enzymes such as aromatic amino acid hydroxylase and nitric oxide synthase (NOS), and it participates in various biological functions (14, 15). As a cofactor of phenylalanine hydroxylase (PAH), BH4 is used clinically as an orphan drug in patients with phenylketonuria $(16,17)$. As a cofactor of tyrosine hydroxylase $(\mathrm{TH})$ and tryptophan hydroxylase, $\mathrm{BH} 4$ regulates the production of various neurotransmitters, such as dopamine, noradrenaline (NA), adrenaline, and serotonin (14). Indeed, congenital BH4 deficiency in human results in abnormal neurological function and causes a dopa-responsive dystonia called Segawa's disease (18, 19). As a cofactor of NOS, and especially endothelial NOS (eNOS), BH4 plays a role in dimer formation of its active and stabilized form to regulate eNOS activity $(20,21)$. Under condition of oxidative stress, such as diabetes or cardiovascular disease, $\mathrm{BH} 4$ is converted to 7,8-dihydrobiopterin ( $\mathrm{BH} 2)$, 
which causes eNOS dysfunction, as $\mathrm{BH} 2$ is inactive for NOS cofactor function, and competes with $\mathrm{BH} 4$ for BH4 binding, referred to as "eNOS uncoupling" $(20,21)$. Indeed, endothelial dysfunction by eNOS uncoupling occurs in patients with cardiovascular disease, and supplementation of BH4 has been examined in clinical trials for the expected vasodilatation caused by $\mathrm{BH} 4$ via NO production that elevates the $\mathrm{BH} 4 / \mathrm{BH} 2$ ratio and leads to recoupling of eNOS (20-22).

Notably, BH4 regulates the contents of enzymatic products, including regulatory factors of BAT, such as NA $(23,24)$ and NO $(25)$. NA stimulation via $\beta_{3}$-adrenergic receptor is well known as a BAT activator $(10,23,24)$. NO, especially eNOS-derived NO, regulates BAT function by induction of mitochondrial biogenesis (25); however, participation of $\mathrm{BH} 4$ in BAT function remains unclear.

In the current study, we investigated whether BH4 activates BAT function and BH4-induced BAT participates in the regulation of systemic energy metabolism. We used hph-1 mice, which exhibit genetic deficiency in GTP-cyclohydrolase I (GTPCH I), a rate-limiting enzyme of de novo BH4 synthesis $(19,26)$. We demonstrate that a mouse model of $\mathrm{BH} 4$ deficiency exhibits obesity, adiposity, glucose intolerance, and insulin resistance; impaired BAT function also was found in these mice. Furthermore, BH4 plays a critical role in the regulation of systemic energy homeostasis and, subsequently, in the regulation of metabolic-related organs. BH4 has an ameliorating effect on BAT dysfunction as well as on metabolic disturbances in BH4-deficient mice. Our present study indicates that BH4 activates BAT function and regulates systemic energy metabolism and has potential as a treatment for metabolic disorders, such as obesity and diabetes.

\section{Results}

Deficiency of BH4 induces adiposity, glucose intolerance, insulin resistance, and disturbance of energy metabolism. We first investigated the role of BH4 in systemic energy metabolism using hph-1 mice $(19,26)$, in which $\mathrm{BH} 4$ synthesis is deficient. Hph-1 mice showed decreased expression of GTPCH I at the protein level (Supplemental Figure 1A; supplemental material available online with this article; https://doi.org/10.1172/jci. insight.91981DS1) and exhibited a marked reduction of BH4 levels in the respective tissues, blood, and urine (Supplemental Figure 1, B-E) compared with control mice of the same background at 6 weeks of age. Hph-1 mice exhibited excessive body weight gain compared with control mice under high-fat diet (HFD) for 4 weeks (Figure 1A). Food intake was not changed in mice under control fat diet (CFD) or HFD (Supplemental Figure 2A). Excessive visceral fat accumulation was found in hph-1 mice compared with control mice under HFD by abdominal CT imaging (Figure 1, B and C). Volume of both epididymal and inguinal white adipose tissues (eWAT and iWAT) was approximately 2-fold increased in hph-1 mice compared with control mice under HFD, and adipose cell size was enlarged (Figure 1, D and E). Liver triglyceride (TG) content was increased in hph-1 mice compared with that of control mice under CFD (Figure 1F), and liver weight (Supplemental Figure 2B) was increased in hph-1 mice under HFD. Liver total cholesterol (T-CHO) content was not changed in mice fed either diet (Figure 1F).

In addition, hph-1 mice exhibited impaired glucose metabolism under both CFD and HFD. Hph-1 mice had higher fasting blood glucose levels (CFD: control, $48.3 \pm 3.0 \mathrm{mg} / \mathrm{dl}$; hph-1, $70.2 \pm 3.0 \mathrm{mg}$ / d1, $P<0.01$ vs. control; HFD: control, $63.2 \pm 6.4 \mathrm{mg} / \mathrm{dl}$; hph-1, $81.0 \pm 2.1 \mathrm{mg} / \mathrm{dl}, P<0.05$ vs. control; Figure 2A) and fed blood glucose levels (CFD: control, $123.4 \pm 4.1 \mathrm{mg} / \mathrm{dl}$; hph-1, $160.5 \pm 5.7 \mathrm{mg} / \mathrm{dl}, P$ $<0.01$ vs. control; HFD: control, $142.6 \pm 5.5 \mathrm{mg} / \mathrm{dl}$; hph-1, $161.6 \pm 5.4 \mathrm{mg} / \mathrm{dl}, P<0.05$ vs. control; Figure $2 \mathrm{~B}$ ). Intraperitoneal glucose tolerance test (IPGTT) data showed that hph-1 mice exhibited glucose intolerance (Figure 2C) under both CFD and HFD; insulin tolerance test data showed that these mice also had insulin resistance (Figure 2D) under HFD. Pyruvate tolerance test data showed that hph-1 mice had enhanced hepatic gluconeogenesis under both CFD and HFD (Supplemental Figure 3, A and B).

Hph-1 mice showed reduced core body temperature (Figure 2E) and reduced energy expenditure, especially during the early dark phase, which indicates impairment of diet-induced thermogenesis (27) (Figure 2F). These findings indicate that hph-1 mice exhibit adiposity, glucose intolerance, insulin resistance, and disturbed energy metabolism.

Deficiency of BH4 induces impairment of BAT function. To confirm that the reduction of core body temperature and energy expenditure in hph-1 mice could be induced by impaired BAT function, we investigated these mice further. To evaluate cold-induced thermogenesis (CIT), we performed a $4^{\circ} \mathrm{C}$ cold tolerance test (CTT). Core body temperature was found to be dramatically decreased in hph-1 mice compared with that in control mice at 6 weeks of age over 5 hours (Figure 3A). A difference in BAT weight was not found between the two groups of mice (Supplemental Figure 4A). On the other hand, observation of BAT using optical microscopy 
A Changes of body weight

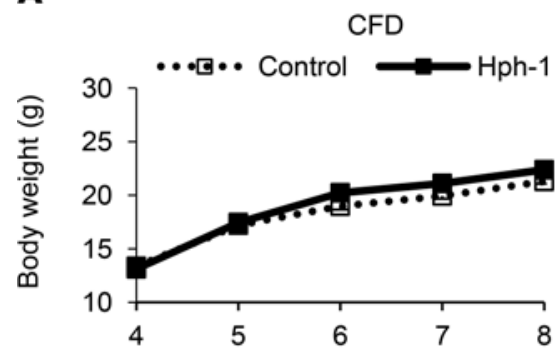

B

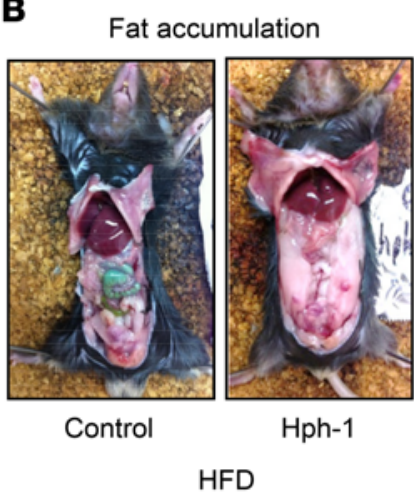

C

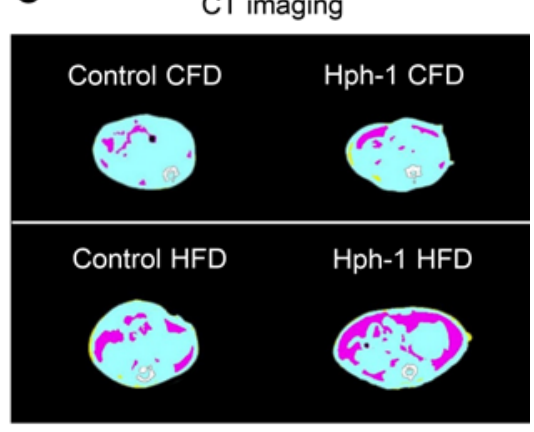

D
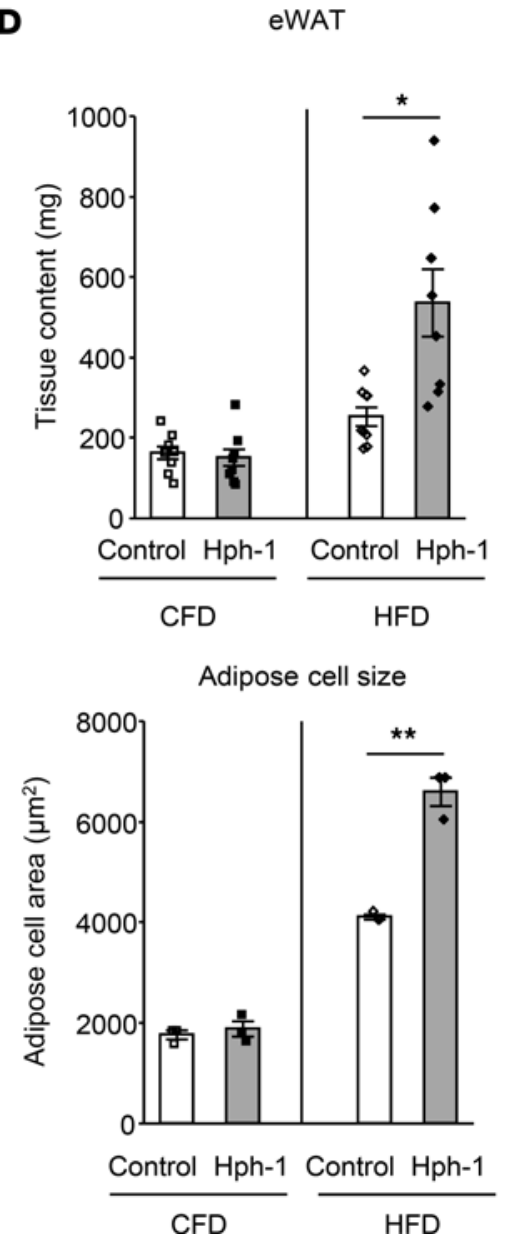

IWAT
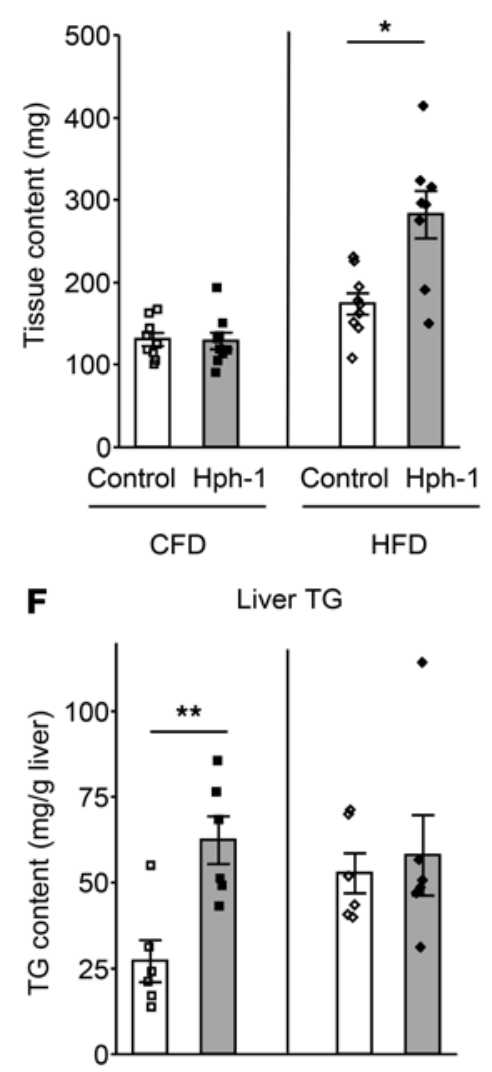

Control Hph-1 Control Hph-1

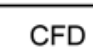

HFD
E
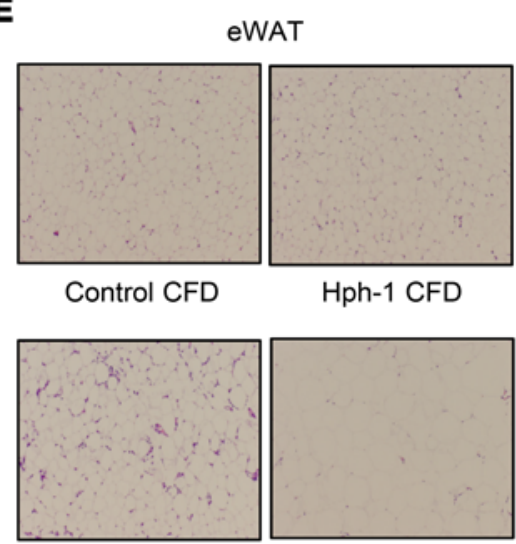

Control HFD

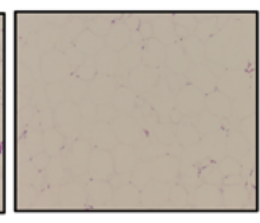

Hph-1 HFD

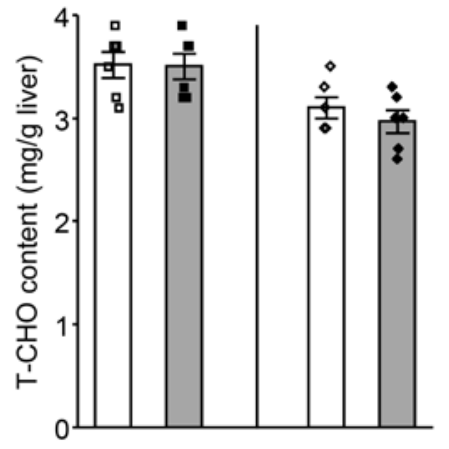

Control Hph-1 Control Hph-1

CFD

HFD

Figure 1. BH4-deficient mice exhibit obesity and adiposity. (A) Body weight changes in hph-1 mice and control mice of the same background under control fat diet (CFD) or high-fat diet (HFD) for 4 weeks from the age of 4 weeks $(n=9)$. (B) Fat accumulation in hph-1 mice and control mice under HFD for 4 weeks. (C-F) CT imaging of adipose distribution (C), fat volume of epididymal and inguinal white adipose tissues (eWAT and iWAT) (D; $n=8-9)$, H\&E staining and white adipose cell size of eWAT $(\mathbf{E} ; n=3$ ) (original magnification, $\times 10$ ), liver triglyceride (TC) and total cholesterol (T-CHO) contents (F; $n=6$ ) under CFD or HFD for 4 weeks. Values are mean \pm SEM. Statistical analysis was performed by 2-way ANOVA with Bonferroni post-hoc test (A) or Student's $t$ test $(\mathbf{D}-\mathbf{F}) .{ }^{*} P<0.05,{ }^{* *} P<0.01$ vs. control group. 
showed increased size of lipid droplets in hph-1 mice compared with that in control mice under CFD (Figure 3B). Furthermore, using transmission electron microscopy, lower mitochondrial density and impairment of cristae structure in mitochondria were observed in BAT from hph-1 mice (Figure 3C). In BAT of hph-1 mice, the expression level of Gch1, a gene coding for GTPCH I, and that of BAT-related genes, including Ucp1, peroxisome proliferator-activated receptor $\gamma$ coactivator-1 (Pgcl $\alpha$ ), and deiodinase, iodothyronine, type II (Dio2), were decreased (Figure 3D). In addition, UCP1 protein expression as well as total amount of UCP1 protein per mouse was decreased (Figure 3E and Supplemental Figure 4, A-C). These data from hph-1 mice indicate that deficiency of BH4 induces impairment of BAT function.

Supplementation of BH4 to BH4-deficient mice ameliorates BAT function as well as metabolic disturbance. To ascertain whether supplementation of $\mathrm{BH} 4$ ameliorates BAT function in this mouse model, we administered $\mathrm{BH} 4$ to hph-1 mice. After administration of $20 \mathrm{mg} \mathrm{BH} 4 / \mathrm{kg}$ body weight in hph-1 mice for 10 days from the age of 5 weeks, core body temperature was increased compared with that in hph-1 mice administered saline (Figure 4A); the size of lipid droplets was reduced and Ucp1 gene expression was increased in BAT of hph-1 mice (Figure 4, B and C). Moreover, after the administration of $\mathrm{BH} 4$, the disturbed glucose metabolism was ameliorated, including higher fasting blood glucose levels (saline, $67.1 \pm 1.8 \mathrm{mg} / \mathrm{dl} ; \mathrm{BH} 4,57.2 \pm 1.4 \mathrm{mg} / \mathrm{dl}$, $P<0.01$ vs. saline; Figure 4D), fed blood glucose levels (saline, $166.6 \pm 7.2 \mathrm{mg} / \mathrm{dl}$; BH4, $151.0 \pm 7.2 \mathrm{mg} / \mathrm{dl}$, $P<0.05$ vs. saline; Figure 4E), and glucose intolerance by IPGTT in hph-1 mice (Figure 4F). These findings indicate that supplementation of BH4 to hph-1 mice ameliorates BAT function as well as glucose metabolism.

$B A T$ has a critical role in the regulation of systemic energy metabolism by BH4. To clarify the contribution of BAT to BH4-induced regulation of energy metabolism, we performed BAT transplantation (28). 0.1 g BAT collected from control mice (BH4-sufficient mice) or hph-1 mice (BH4-deficient mice) was transplanted into the visceral fat region (bilateral eWAT) of hph-1 mice at 8 weeks of age; they were housed with HFD for 20 days. We then evaluated effects of the transplanted BAT from recipient mice on systemic energy metabolism from the two groups of donor mice. Transplantation of BAT from control mice ameliorated BAT-related functions, including core body temperature after 10 and 20 days, and CIT after 20 days compared with the levels in hph-1 mice (Figure 5, A and B). These data indicate that transplantation of BAT from control mice ameliorated adaptive thermogenesis, including diet-induced thermogenesis and CIT.

Interestingly, BAT transplantation from control mice also resulted in beneficial changes in systemic metabolism and other metabolic-related organs. Excessive weight gain was prevented by BAT transplantation from control mice compared with that from hph-1 mice (Figure 5C). Adiposity evaluated by abdominal CT imaging was decreased and volume of both eWAT and iWAT was decreased by approximately one-half by BAT transplantation from control mice in comparison with those from hph-1 mice; there was also a reduction of adipose cell size (Figure 5, D-G). In addition, impaired glucose metabolism, as shown by higher fed blood glucose levels (BAT transplantation after 10 days: from hph-1 mice, $167.2 \pm 4.6 \mathrm{mg} / \mathrm{dl}$; from control mice, $144.5 \pm 4.9 \mathrm{mg} / \mathrm{dl}, P<0.05$ vs. BAT transplantation from hph-1 mice; BAT transplantation after 20 days: from hph-1 mice, $165.1 \pm 8.6 \mathrm{mg} / \mathrm{dl}$; from control mice, $132.8 \pm 5.0 \mathrm{mg} / \mathrm{dl}, P<0.01 \mathrm{vs}$. BAT transplantation from hph-1 mice; Figure 6A), glucose intolerance by IPGTT (Figure 6B), and insulin resistance (Figure 6C), was ameliorated in BAT transplantation from control mice compared with that in hph-1 mice. In liver tissue, enhancement of gluconeogenesis (Figure 6D), lipid accumulation (Figure 6E), and liver weight (Figure 6F) were reduced and lipid profiles were improved after the BAT transplantation from control mice compared with that from hph-1 mice (plasma TG: BAT transplantation from hph-1 mice, $116.9 \pm 19.1 \mathrm{mg} / \mathrm{dl}$; from control mice, $45.7 \pm 3.7 \mathrm{mg} / \mathrm{dl}, P<0.05$ vs. BAT transplantation from hph-1 mice; Figure $6 \mathrm{G})$. Food intake was not changed in either group of mice (Supplemental Figure 5). These findings clearly indicate that BAT has a central role in $\mathrm{BH} 4$-induced regulation of systemic energy metabolism.

$N A$ participates in the regulation of $B A T$ function by $B H 4$. We then investigated the mechanism of regulation of BAT function by $\mathrm{BH} 4 . \mathrm{BH} 4$ acts as a cofactor of $\mathrm{TH}$, a rate-limiting enzyme of NA synthesis. NA levels were found to be decreased by almost $60 \%$ in BAT and urine, which reflects systemic NA levels, in hph-1 mice compared with control mice (Figure 7, A and B). To evaluate the effect of BH4 on NA production, we used PC12 cells, a cell line derived from pheochromocytoma with a high capacity for catecholamine synthesis (29). Depletion of BH4 by 2,4-diamino-6-hydroxypyrimidine (DAHP), a GTPCH I inhibitor, reduced DOPA production, which correlated with the capacity of catecholamine biosynthesis, including NA, dose-dependently in PC12 cells (Figure 7C). On the other hand, supplementation of BH4 increased DOPA levels in PC12 cells (Figure 7D). These findings support the view that BH4 stimulates NA production and that $\mathrm{BH} 4$ has activating effects on BAT via NA synthesis. 
A Fasting blood glucose
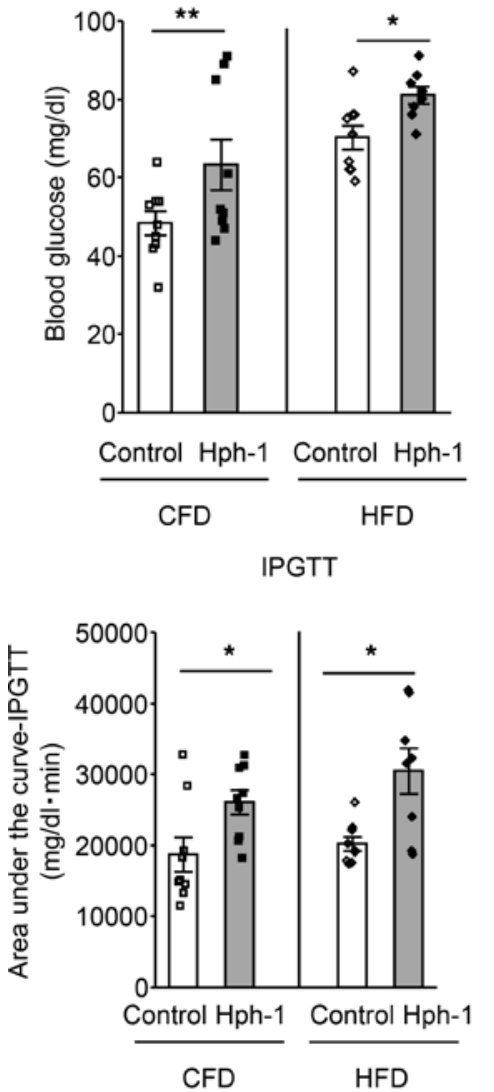

E Core body temperature

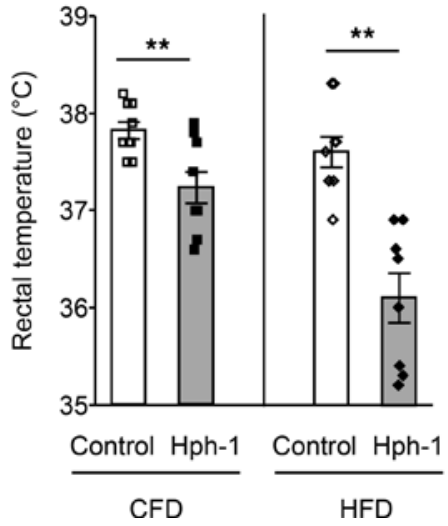

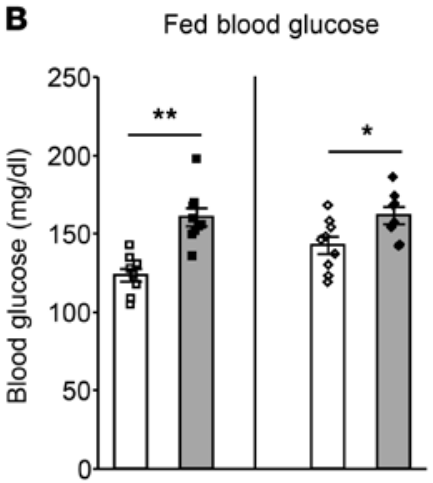

Control Hph-1 Control Hph-1 HFD

D
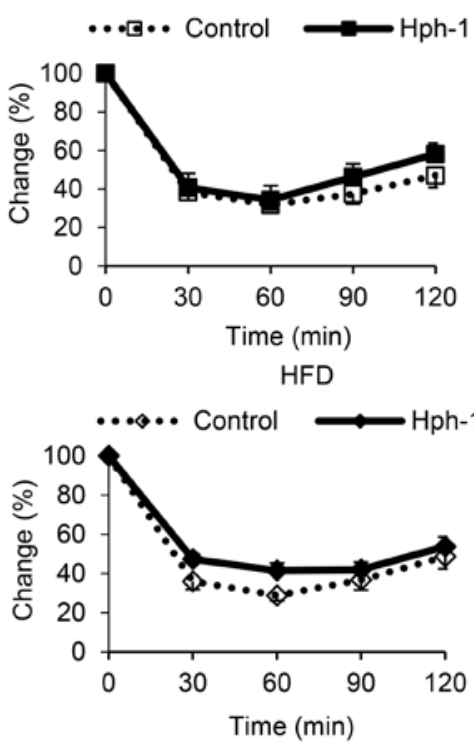

$\mathbf{F}$$$
\text { के }
$$

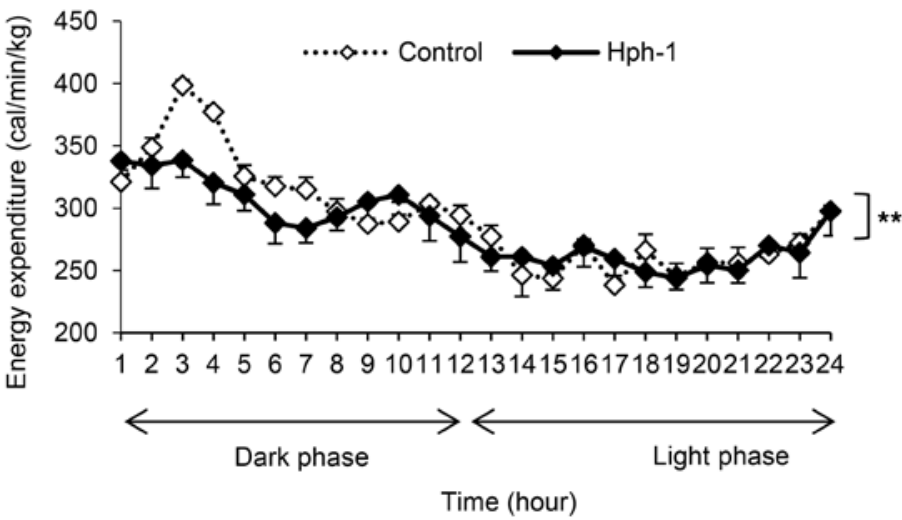

Figure 2. BH4-deficient mice exhibit glucose intolerance, insulin resistance, and disturbance of energy metabolism. (A-E) Fasting blood glucose levels after $16 \mathrm{~h}$ fasting (A; $n=8-9)$, fed blood glucose levels (B; $n=8-9)$, intraperitoneal glucose tolerance test (IPGTT; $1 \mathrm{~g} / \mathrm{kg}$ glucose) after $16 \mathrm{~h}$ fasting (C; $n=$ 8-9), insulin tolerance test (ITT; 1 unit/kg regular insulin) after $6 \mathrm{~h}$ fasting $(\mathbf{D} ; n=8-9)$, and rectal temperature $(\mathbf{E} ; \mathbf{n}=9)$ under control fat diet (CFD) or high fat diet (HFD) for 4 weeks. (F) Energy expenditure during $24 \mathrm{~h}$ under HFD for 4 weeks $(n=4)$. Values are mean \pm SEM. Statistical analysis was performed by 2-way ANOVA with Bonferroni post-hoc test (C, $\mathbf{D}$, and $\mathbf{F})$ or Student's $t$ test $(\mathbf{A}-\mathbf{E}) .{ }^{*} P<0.05,{ }^{* *} P<0.01$ vs. control group. 
NO participates in the regulation of $\mathrm{BAT}$ function by $\mathrm{BH} 4 \mathrm{BH} 4$ also is known to act as a cofactor of eNOS $(20,21)$. NO content was found to be decreased by approximately $60 \%$ in BAT of hph- 1 mice compared with that of control mice (Figure 8A). NO production in BAT following an intraperitoneal single administration of $\mathrm{BH} 4$ was increased almost 2 -fold in hph-1 mice compared with that in hph1 mice administered saline (Figure $8 \mathrm{~B}$ ). These data strongly suggest that deficiency of BH4 induces impairment of $\mathrm{NO}$ synthesis and that $\mathrm{BH} 4$ regulates $\mathrm{NO}$ synthesis in BAT.

$\mathrm{BH} 4$ is converted to $\mathrm{BH} 2$, which causes impairment of dimerization of eNOS and its action $(20,21)$. Hph-1 mice are known to be a model with a reduced $\mathrm{BH} 4 / \mathrm{BH} 2$ ratio, a marker of eNOS uncoupling (21, 30 ), as well as reduced BH4 levels (31) (Supplemental Figure 6A). In addition, anti-nitrotyrosine staining, a marker of eNOS uncoupling, in aortas in the diabetic state following streptozotocin injection also was increased in hph-1 mice compared with that in control mice (Supplemental Figure 6B). To investigate whether the eNOS coupling rate participates in BAT function of hph-1 mice, we performed experiments that cause changes in the $\mathrm{BH} 4 / \mathrm{BH} 2$ ratio by using methotrexate (MTX) (31), an inhibitor of dihydrofolate reductase (DHFR), which converts $\mathrm{BH} 2$ to $\mathrm{BH} 4$. MTX markedly reduced the $\mathrm{BH} 4 / \mathrm{BH} 2$ ratio, decreased core body temperature, and increased blood glucose level (Supplemental Figure 7, A-C), all of which are correlated with the $\mathrm{BH} 4 / \mathrm{BH} 2$ ratio (Supplemental Figure 7, D and $\mathrm{E}$ ). Previous findings suggested that $\mathrm{TH}$ activity is regulated only by the $\mathrm{BH} 4$ level, regardless of the $\mathrm{BH} 2$ level, whereas eNOS activity is regulated mainly by the $\mathrm{BH} 4 / \mathrm{BH} 2$ ratio $(21,30)$. Thus, the present findings support the view that eNOS-derived NO may take part in the regulation of BH4-induced BAT function. To determine whether $\mathrm{BH} 4$ regulates BAT function through eNOS exclusively, we used eNOS-deficient mice. However, administration of BH4 to eNOS-deficient mice had a preventive effect on the reduction of core body temperature induced by CTT (Supplemental Figure 8, A and B). These data indicate that BH4-mediated BAT function is not mediated by eNOS alone; the collaborative involvement of other regulatory factors, such as NA, is implicated.

BH4 directly activates brown adipocytes via NO. We then investigated whether $\mathrm{BH} 4$ directly regulates BAT in brown adipocytes. BH4 increased expression of BAT-related genes, including Ucp1, Pgc1a, and Dio2, as well as protein expression of UCP1 dose-dependently after 24-hour incubation with brown adipocytes (Figure 9, A and B). The mitochondrial respiration rate in total was increased by $\mathrm{BH} 4$. The level of proton leak was increased, which can lead to elevation of the uncoupling rate, suggesting that $\mathrm{BH} 4$ may have beneficial effects on the physiological characteristics of brown adipocytes (Figure 9, C-E). BH4 was found to directly increase NO production in brown adipocytes dose-dependently (Figure 9F). NG-nitro-L-arginine methyl ester (L-NAME), a NOS inhibitor, cancelled the enhancement of Ucp1 expression by BH4 (Figure 9G). These data clearly suggest that BH4 directly affects brown adipocytes in the activation of UCP1-mediated thermogenic function NO-dependently. NOS has three isoforms: eNOS, neuronal NOS, and inducible NOS (32). To investigate whether $\mathrm{BH} 4$ increases $\mathrm{NO}$ content in BAT via eNOS, BH4 was administered in eNOS-deficient mice. Although the single administration of $\mathrm{BH} 4$ to wild-type mice increased NO production in BAT (Supplemental Figure 9A), the increase in NO production did not occur in BAT of eNOS-deficient mice (Supplemental Figure 9B). These findings support the view that BH4 activates brown adipocytes eNOS-dependently.

\section{Discussion}

BAT is a central organ that increases energy expenditure and has a remarkable capacity to dissipate energy and produce heat via UCP1 by consuming free fatty acids and glucose (10). In recent decades, it has been reported that the amount of BAT detected using fluorodeoxyglucose-PET and X-ray CT is inversely correlated with BMI and glucose levels in adult humans (6-9), suggesting a role of BAT in energy expenditure as well as systemic metabolism. Moreover, in recent studies, it has been shown that BAT transplantation has antiobesity effects as well as glucose-lowering effects in diet-induced obesity model mice $(28,33)$, supporting the view that BAT is involved in the regulation of systemic energy and glucose metabolism. Thus, numerous studies, including human research, suggest that BAT is a potentially novel therapeutic target for metabolic diseases such as obesity and diabetes.

In the present study, we show for the first time to our knowledge that $\mathrm{BH} 4$ acts as a major regulating factor of BAT function and that BH4-induced BAT has a critical role in the regulation of systemic energy homeostasis and, subsequently, in the regulation of metabolic-related organs. The proposed mechanism by which BAT function is regulated by BH4 is summarized in Supplemental Figure 10. BH4 is an essential coenzyme of $\mathrm{TH}$, which is a rate-limiting enzyme of catecholamine biosynthesis. Owing to the limited supply of $\mathrm{BH} 4$ for the $\mathrm{TH}$ system, the $\mathrm{BH} 4$ level ultimately regulates the monoamine production such as that 
A

Cold tolerance test

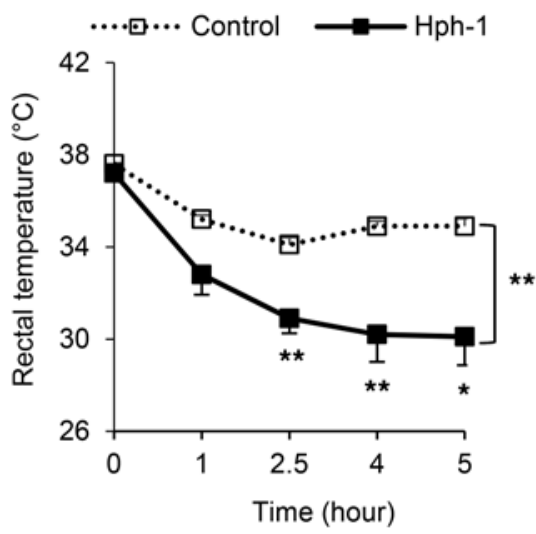

C

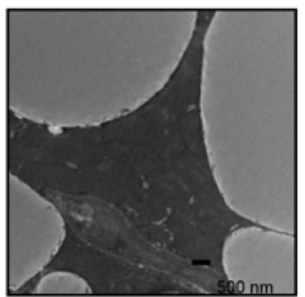

BAT

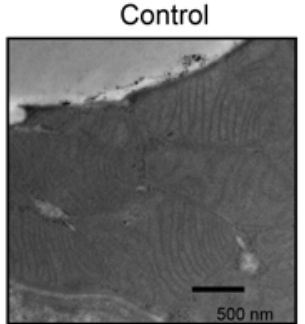

Control

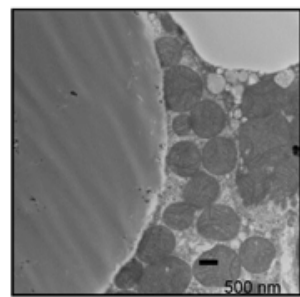

Hph-1

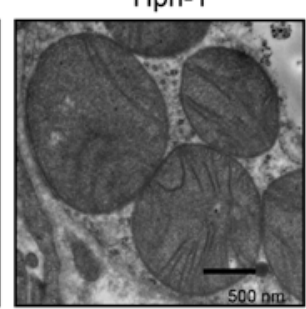

Hph-1
E UCP1 expression

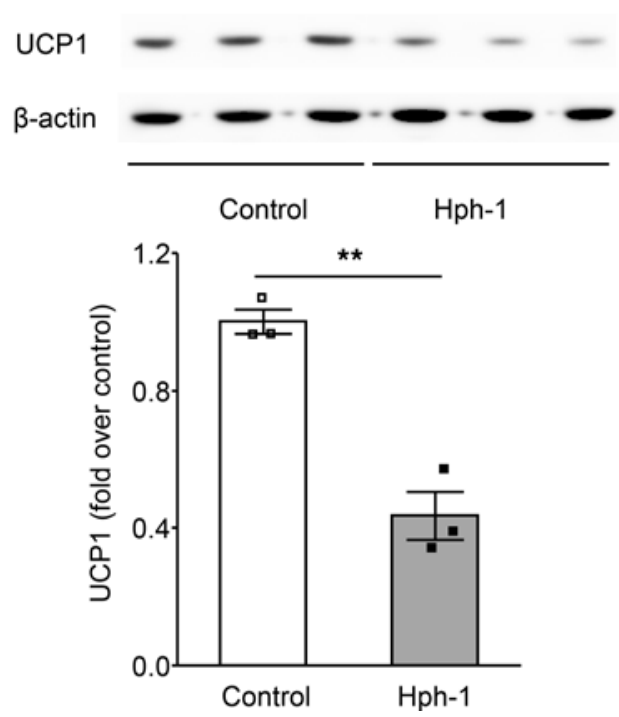

BAT

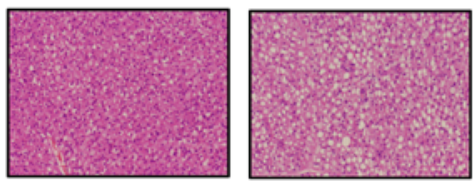

Hph-1

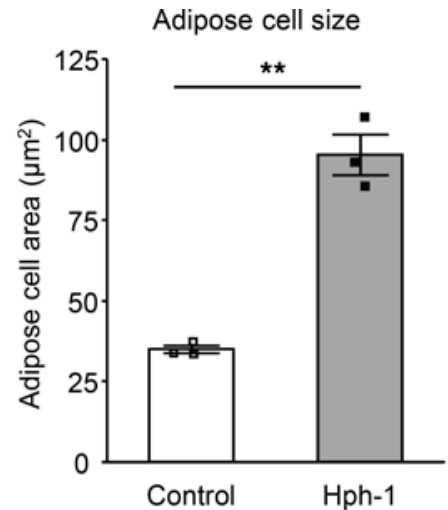

BAT-related gene expression

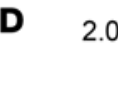

$\square$ Control $\square \mathrm{Hph}-1$

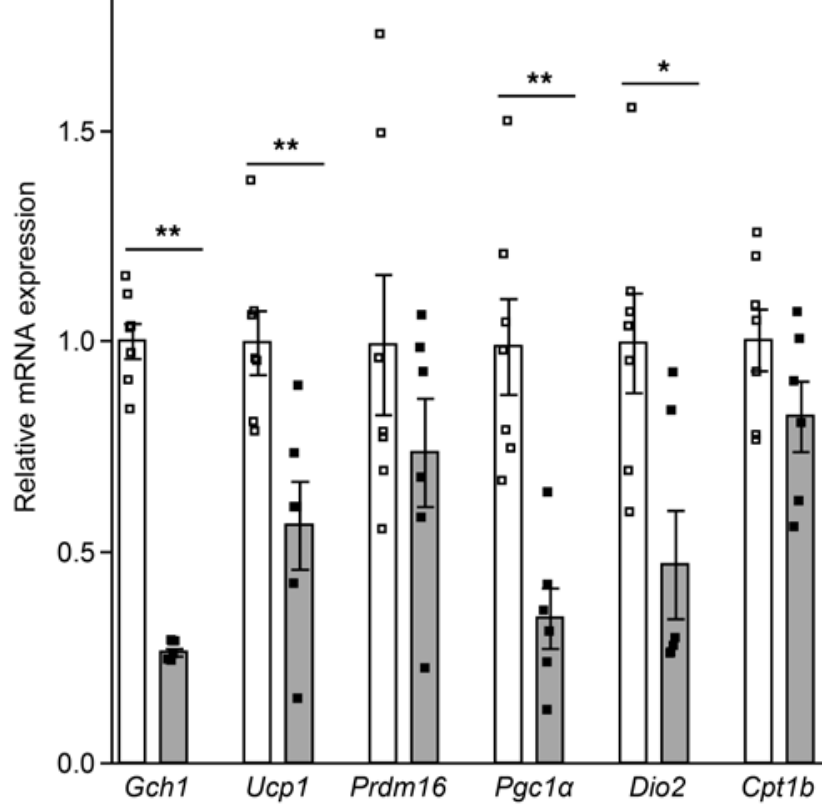

Figure 3. Impairment of BAT occurred in BH4-deficient mice. Changes of rectal temperature in $4^{\circ} \mathrm{C}$ cold tolerance test $(A ; n=7-8), H \& E$ staining and white adipose cell size of brown adipose tissue (BAT) $(\mathbf{B} ; n=3$ ) (original magnification, $\times 15)$, mitochondrial morphology in BAT by electron microscopy (C) (original magnification, $\times 5,000$ [top], ×15,000 [bottom]; scale bar: $500 \mathrm{~nm})$, mRNA levels by quantitative RT-PCR (D; $n=6-7)$, and protein expression levels of UCP1 $(E ; n=3)$ in BAT of hph-1 mice and control mice of the same background at 6 weeks of age under control fat diet. Values are mean \pm SEM. Statistical analysis was performed by 2-way ANOVA with Bonferroni post-hoc test (A) or Student's $t$ test (B, D, and E). ${ }^{*} P<0.05,{ }^{* *} P<0.01$ vs. control group.

of NA in sympathetic nerve endings as well as the nerve cell $(14,34,35)$. NA release promoted by $\mathrm{BH} 4$ acts on adrenergic receptors, primarily the $\beta_{3}$-adrenoceptor, in BAT and activates BAT function $(36,37)$. BH4 also works as a cofactor of eNOS and promotes NO biosynthesis $(20-22,38)$. eNOS is known to be highly expressed in vascular tissues (39), and BAT in vascular-rich organs is well characterized (40). Continuous 
A Core body temperature

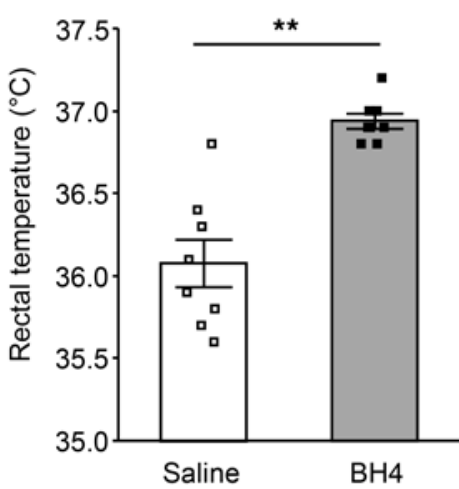

C

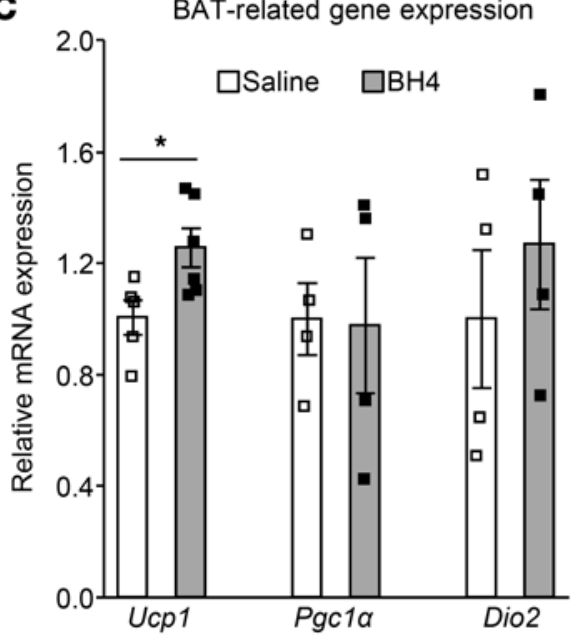

$\mathbf{F}$

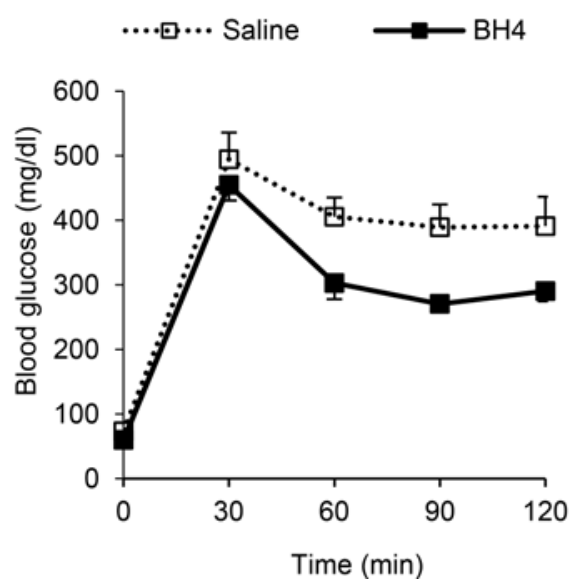

B

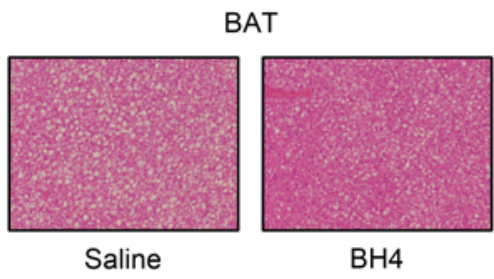

D Fasting blood glucose

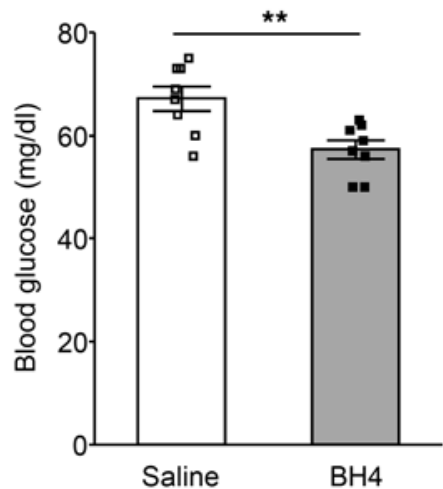

IPGTT

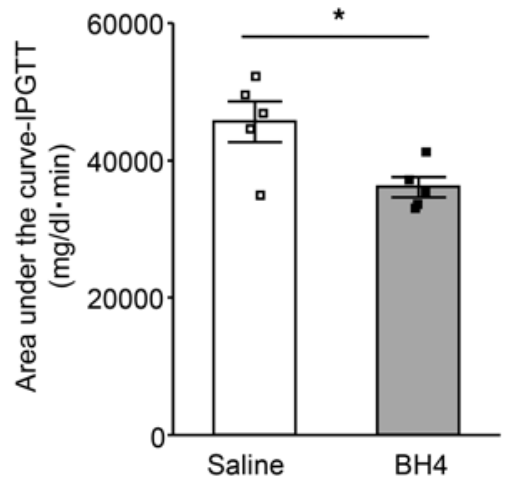

Figure 4. Supplementation of BH4 to BH4-deficient mice ameliorates BAT dysfunction as well as metabolic disturbance. Rectal temperature (A; $n=8)$, H\&E staining and white adipose cell size of brown adipose tissue (BAT) $(\mathbf{B} ; n=3)$ (original magnification, $\times 15)$, mRNA levels in BAT by quantitative RT-PCR (C; $n=4-6)$, fasting blood glucose levels after 16 hours fasting $(\mathbf{D} ; n=8)$, fed blood glucose levels $(\mathbf{E} ; n=8)$, and intraperitoneal glucose tolerance test (IPGTT; $2 \mathrm{~g} / \mathrm{kg}$ glucose) after 16 hours fasting $(\mathbf{F} ; n=5)$ in hph-1 mice after the treatment with or without $20 \mathrm{mg}$ tetrahydrobiopterin (BH4)/kg body weight for 10 days from the age of 5 weeks. Values are mean \pm SEM. Statistical analysis was performed by 2-way ANOVA with Bonferroni post-hoc test (F) or Student's $t$ test $(\mathbf{A}-\mathbf{F}) .{ }^{*} P<0.05,{ }^{* *} P<0.01$ vs. saline group. 
A Core body temperature (Post 10 days)

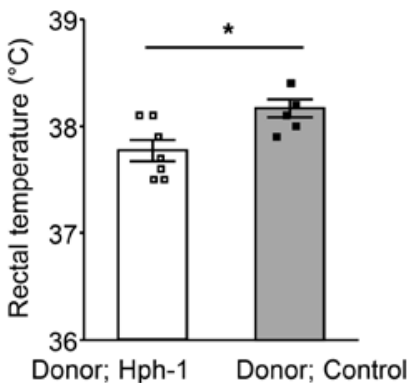

Recipient; Hph-1 Recipient; Hph-1
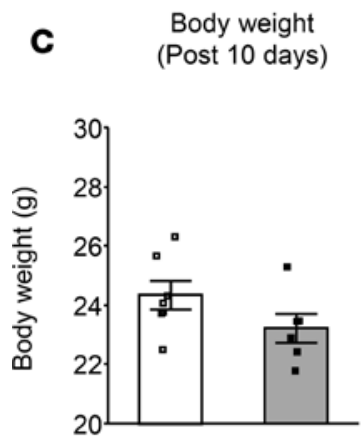

Donor; Hph-1 Donor; Control Recipient; Hph-1 Recipient; Hph-1

E CT imaging

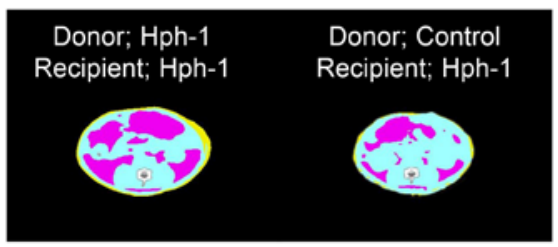

G

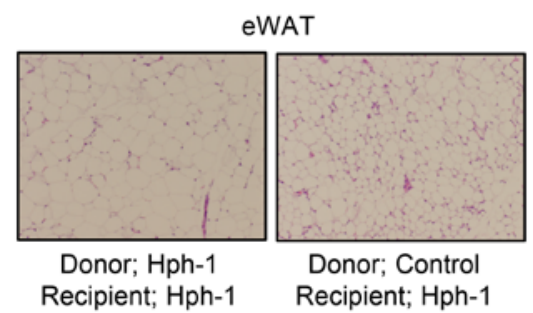

Core body temperature

(Post 20 days)

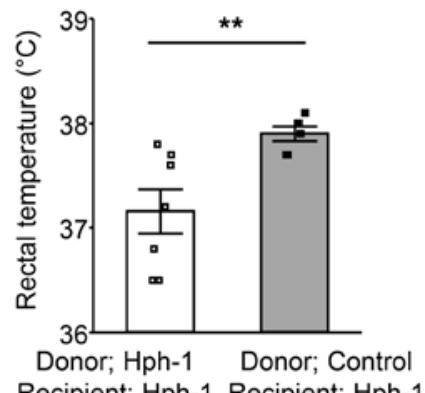

Body weight

(Post 20 days)

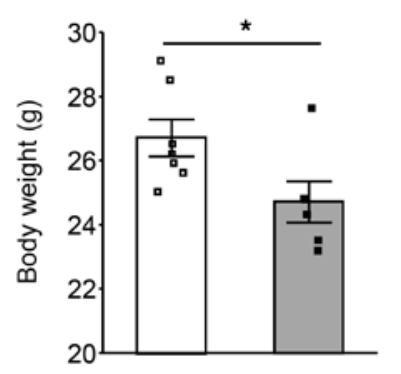

Donor; Hph-1 Donor; Control Recipient; Hph-1 Recipient; Hph-1

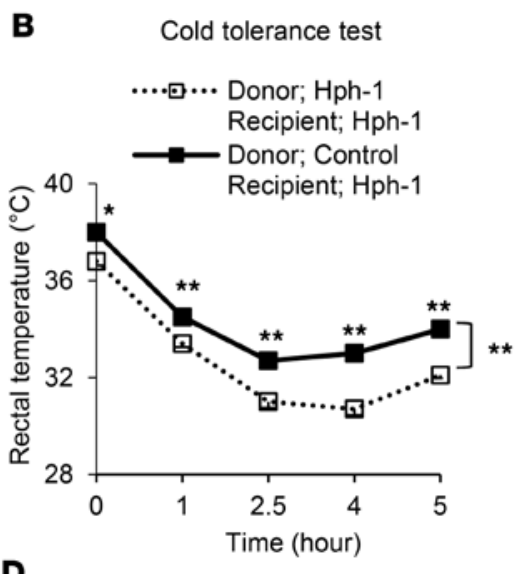

D

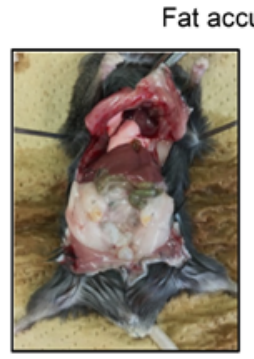

Donor; Hph-1 Recipient; Hph-1

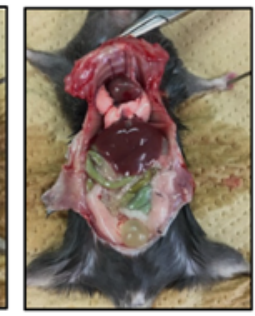

Donor; Control Recipient; Hph-1
F
eWAT

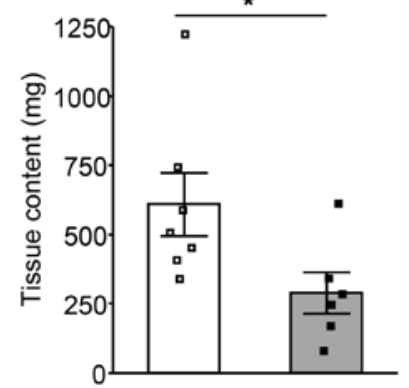

Donor; Hph-1 Donor; Control

Recipient; Hph-1 Recipient: Hph-1 Recipient; Hph-1 Recipient; Hph-1
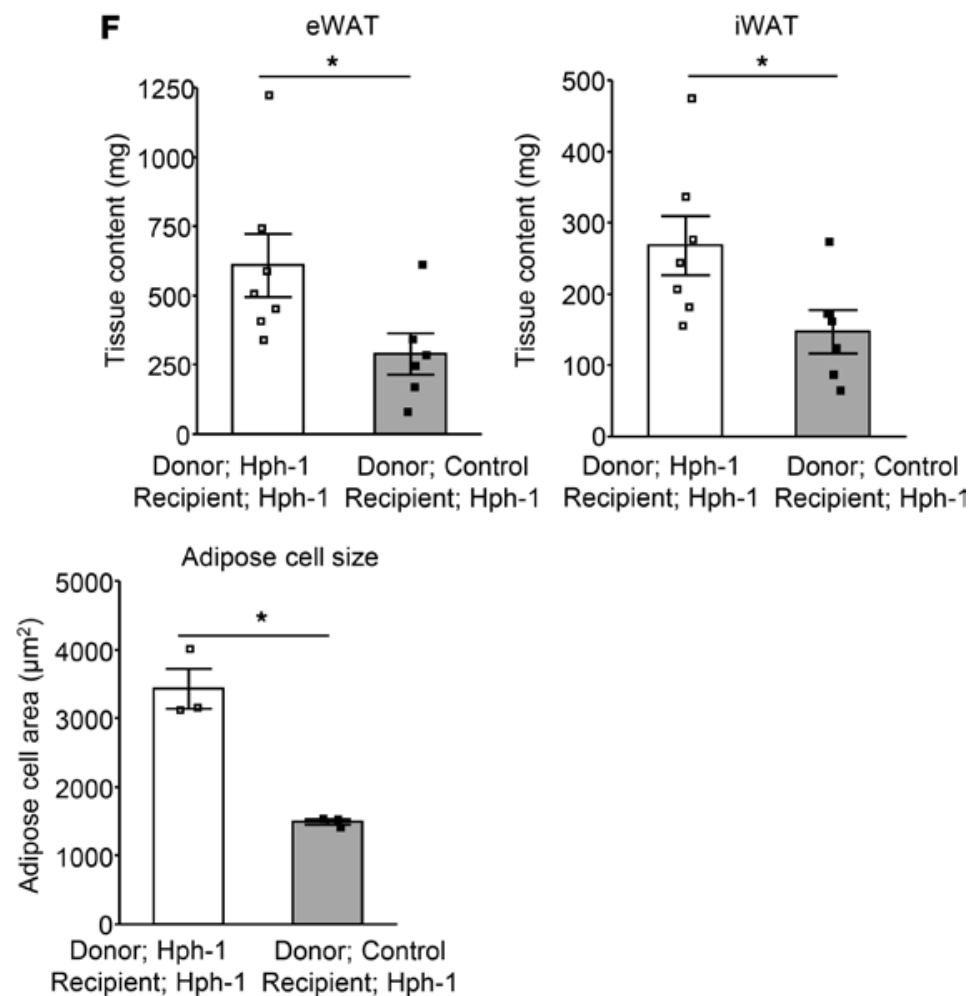

Figure 5. BAT transplantation derived from BH4-sufficient mice improves BAT function and ameliorates adiposity in BH4-deficient mice. (A-C) Rectal temperature (A; $n=6-7), 4^{\circ} \mathbf{C}$ cold tolerance test (B; $\left.n=5-7\right)$, body weight gains (C; $\left.n=6-7\right)$, fat accumulation (D), computed tomography (CT) imaging of adipose distribution (E), fat volume of epididymal and inguinal white adipose tissues (eWAT and iWAT) (F; $n=6-7$ ), and H\&E staining and adipose cell size in eWAT $(\mathbf{G} ; n=3$ ) (original magnification, $\times 10$ ) in hph-1 mice transplanted $0.1 \mathrm{~g}$ brown adipose tissue (BAT) derived from hph-1 mice (BH4-deficient mice) or control mice of the same background (BH4-sufficient mice) under high fat diet for 20 days from the age of 8 weeks. Values are mean \pm SEM. Statistical analysis was performed by 2 -way ANOVA with Bonferroni post-hoc test (B) or Student's $t$ test $(\mathbf{A}, \mathbf{C}, \mathbf{F}$, and $\mathbf{G}) .{ }^{*} P<0.05$, ${ }^{* *} P<0.01$ vs. hph-1 mice transplanted BAT derived from hph-1 mice. 
high exposure of $\mathrm{NO}$ around BAT by BH4 induces NO uptake by diffusion in BAT, which leads to promotion of mitochondrial biogenesis and capacity, which includes energy dissipation and heat production (25, $41,42)$. It is known that eNOS is expressed abundantly in brown adipocytes $(25,42)$, and we have found that $\mathrm{BH} 4$ directly affects brown adipocytes by increasing UCP1 expression and the thermogenic response via NO. Thus, the current study shows that BH4 cooperatively activates BAT function via both NA and NO.

We also demonstrate that $\mathrm{BH} 4$ regulates systemic energy metabolism via BAT activation and that these effects spread to glucose and lipid metabolism as well as to other metabolic organs, such as liver and white adipose tissue (Supplemental Figure 10). We show here that BH4-deficient mice (hph-1 mice) exhibit adiposity, glucose intolerance, and insulin resistance derived from BAT dysfunction. Supplementation of BH4 ameliorates this BAT dysfunction as well as that of glucose dysmetabolism. Moreover, in our experiment using an applied BAT transplantation technique, we found that BAT transplantation from BH4-sufficient mice ameliorated adiposity, glucose intolerance, and insulin resistance in BH4-deficient mice. Thus, hph-1 mice, which exhibit genetic deficiency in GTPCH I, a rate-limiting enzyme of de novo BH4 synthesis, are useful as a model of deficiency in BAT function for elucidation of the detailed role of BAT.

Consistent with previous studies $(28,33,43)$, we demonstrate strong ameliorating effects on metabolic disturbance by BAT transplantation. This is the first report to our knowledge that BAT transplantation affects metabolic regulation using an animal model of BAT dysfunction. Notably, the effects on core body temperature, body weight, and blood glucose levels all appear simultaneously after only 10 days and sooner compared with previous studies. For instance, Liu et al. reported that the body weight gain was suppressed 3 weeks after BAT transplantation in $o b / o b$ mice (43). Moreover, Stanford et al. reported that improved glucose tolerance, increased insulin sensitivity, lower body weight, and decreased fat mass were found in HFD-induced obese mice by 8 to 12 weeks following BAT transplantation (28). In contrast with our study, these reports did not use a BAT-deficient model. As we performed BAT transplantation using these model mice, the effects of BAT transplantation in the amelioration of metabolic dysfunction might occur more rapidly and strongly. These results suggest that $\mathrm{BH} 4$-induced BAT regulates the thermogenic response as well as systemic energy homeostasis, including hepatic gluconeogenesis and steatosis and visceral and subcutaneous fat accumulation by BH4. Our findings support the view that BH4-induced BAT activation has the potential to ameliorate metabolic disturbance.

Regarding possible mechanisms of the influence of systemic metabolism by BAT transplantation, it should first be considered that increased heat production and energy expenditure may well participate in the subsequent metabolic effects. Second, it is likely that BAT transplanted into the visceral cavity of BH4-deficient mice from BH4-sufficient mice will utilize free fatty acids and glucose from the visceral fat area and, thus, contribute to rapid amelioration of metabolic disturbance. Regarding the area of the BAT graft, Stanford et al. reported that, compared with these beneficial effects of BAT transplantation into the visceral cavity, transplantation of BAT into the subcutaneous cavity of mice did not result in changes in glucose tolerance (28). However, there have been reports that beneficial metabolic effects were obtained by BAT transplantation into the subcutaneous region $(33,43)$. Further investigation is required to elucidate of the regional regulation of transplanted BAT. Regarding the regulatory factors of systemic energy metabolism by BAT transplantation, participation of secreted factors, such as fibroblast growth factor 21 (FGF21), IL-6, and insulin-like growth factors 1 (IGF-1), has been proposed $(28,43,44)$. While we did not investigate these factors in the present study, it is also possible that $\mathrm{BH} 4$ itself or $\mathrm{BH} 4$-induced NO and NA participate in the beneficial effects on metabolism after the BAT transplantation. However, it is unlikely that BH4 itself secreted from transplanted BAT exerts the metabolic effects in the absence of BAT, as the volume of BH4 secretion is very subtle in adipose tissue compared with that of other tissues, such as liver (45). Herein, it could be thought that BAT activated by $\mathrm{BH} 4$ contributes to an increase in energy expenditure and nutrient utilization and ameliorates metabolic abnormalities in hph-1 mice.

We also investigated the participation of NA and NO in the regulation of BAT by BH4. Regarding NO, Nisoli et al. showed that eNOS is one of the regulatory factors of BAT by using eNOS-deficient model mice (25). The function of eNOS is regulated by many factors, such as mRNA expression of eNOS, L-arginine, influx of $\mathrm{Ca}^{2+}$, and $\mathrm{BH} 4(21,39,46)$. Among these, we show here that $\mathrm{BH} 4$ has an important role in eNOS-derived BAT regulation. BH4 plays a role in dimer formation of the active and stabilized form of eNOS $(20,21)$. BH4 is converted to $\mathrm{BH} 2$ by exposure to oxidative stress, such as diabetes and obesity (47-49). We have shown that the value of the $\mathrm{BH} 4 / \mathrm{BH} 2$ ratio, which reflects the extent of eNOS uncoupling, is strongly correlated with BAT functions such as thermogenesis and glucose tolerance. In fact, hph-1 
A Fed blood glucose (Post 10 days)

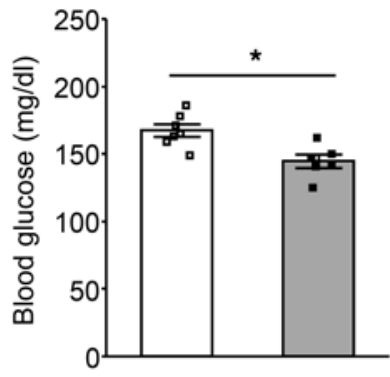

Donor; Hph-1 Donor; Control Recipient; Hph-1 Recipient; Hph-1
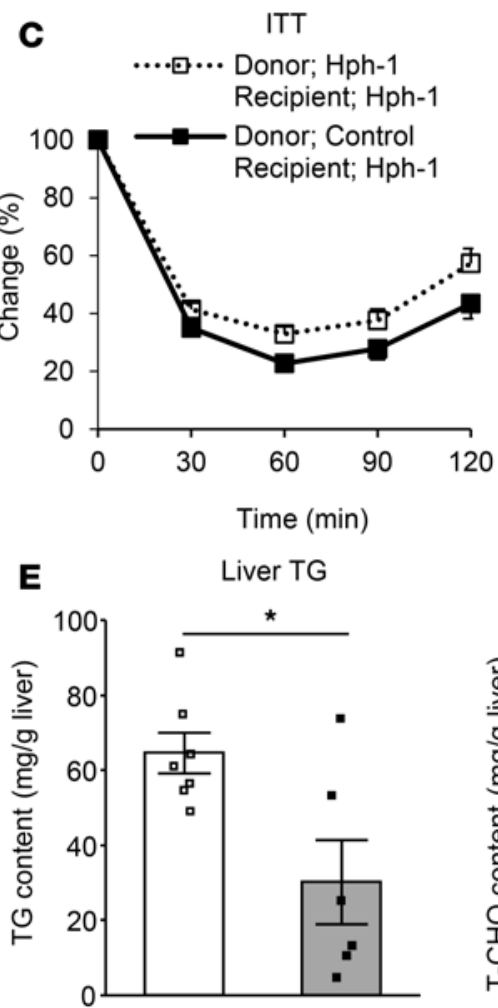

Donor; Hph-1 Donor; Control Recipient; Hph-1 Recipient; Hph-1

$\mathbf{F}$

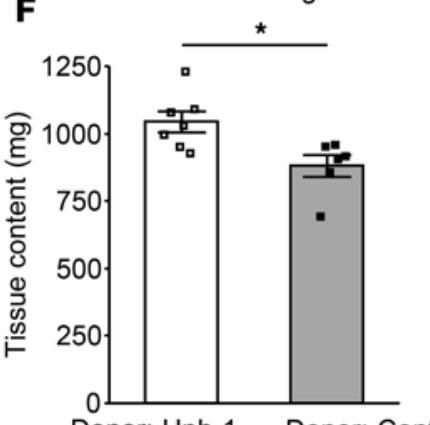

Donor; Hph-1 Donor; Control Recipient; Hph-1 Recipient; Hph-1
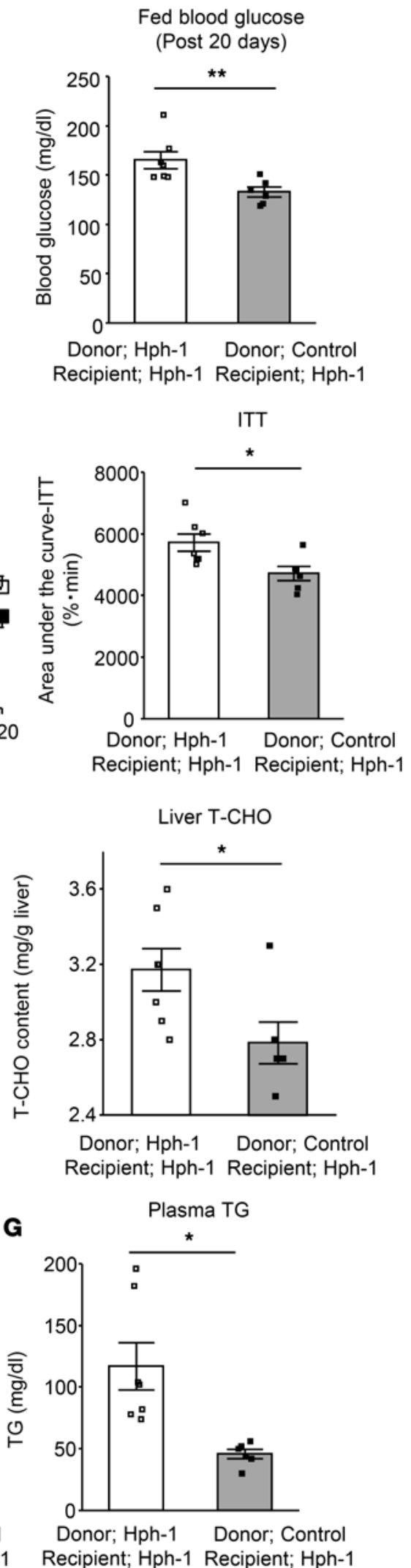

B IPGTT

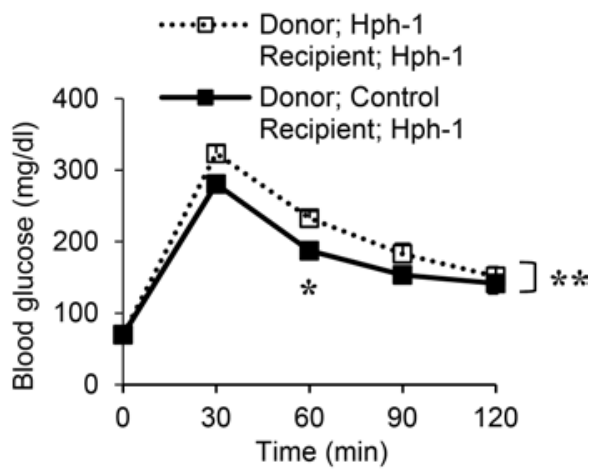

D

PTT

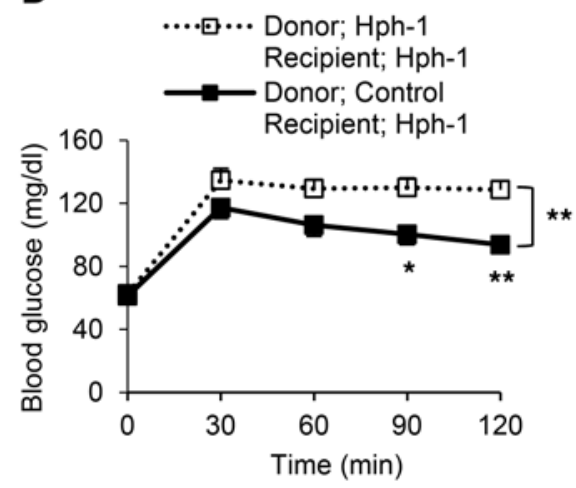

Figure 6. BAT transplantation derived from BH4-sufficient mice improves BAT function as well as ameliorates glucose intolerance and insulin resistance in BH4-deficient mice. Fed blood glucose levels (A; $n=6-7)$, intraperitoneal glucose tolerance test (IPGTT; $1 \mathrm{~g} / \mathrm{kg}$ glucose) after 16 hours fasting (B; $n=6-7$ ), insulin tolerance test (ITT; 1 unit/ $\mathrm{kg}$ regular insulin) after 6 hours fasting $(\mathbf{C} ; n=$ 6-7), pyruvate tolerance test (PTT; $1 \mathrm{~g} / \mathrm{kg}$ pyruvate) after 16 hours fasting (D; $n=6-7$ ), liver triglyceride (TC) and total cholesterol (T-CHO) content (E; $n=6-7)$, liver weight $(\mathbf{F} ; n=6-7)$, and plasma TG concentration (G; $n=6-7)$ in hph-1 mice transplanted with $0.1 \mathrm{~g}$ brown adipose tissue (BAT) derived from hph-1 mice (BH4-deficient mice) or control mice of the same background (BH4-sufficient mice) under high-fat diet for 20 days from the age of 8 weeks. Values are mean \pm SEM. Statistical analysis was performed by 2-way ANOVA with Bonferroni post-hoc test (B-D) or Student's $t$ test (A, C, E-G). ${ }^{*} P<0.05,{ }^{* *} P<$ 0.01 vs. hph-1 mice transplanted BAT derived from hph-1 mice. 
A
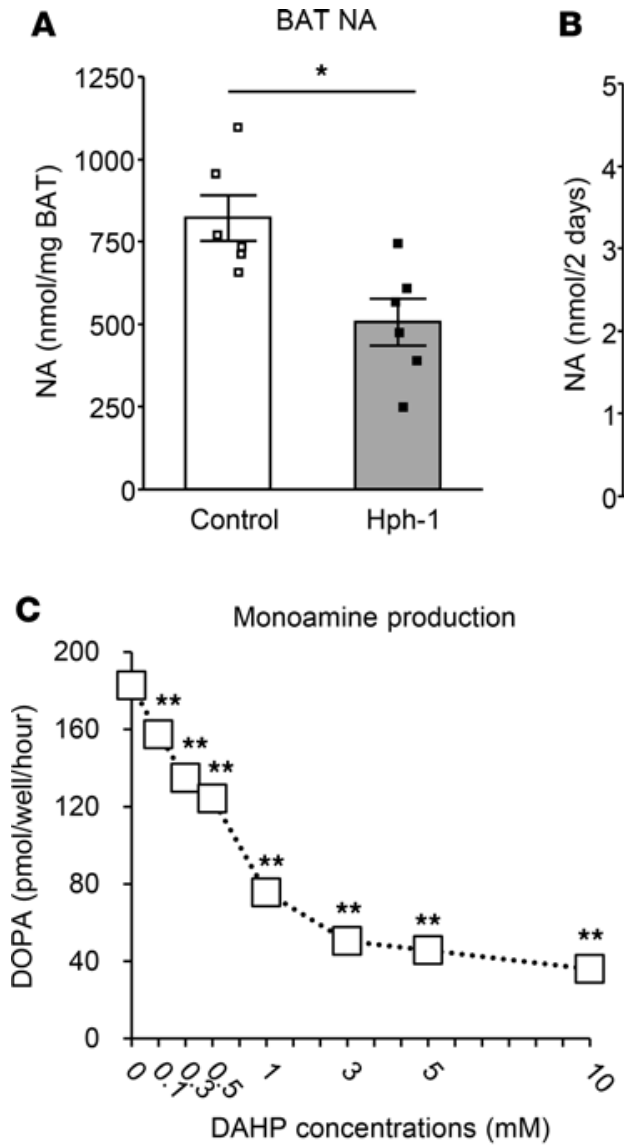

B

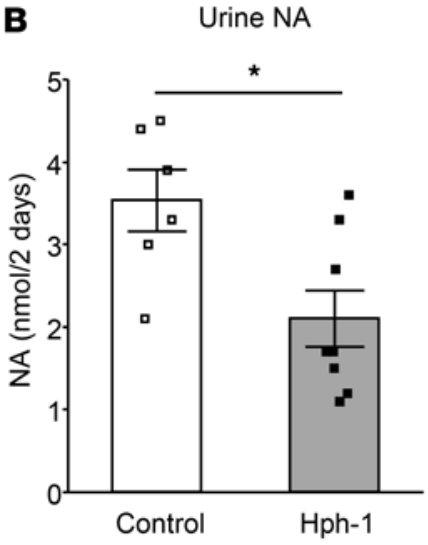

D

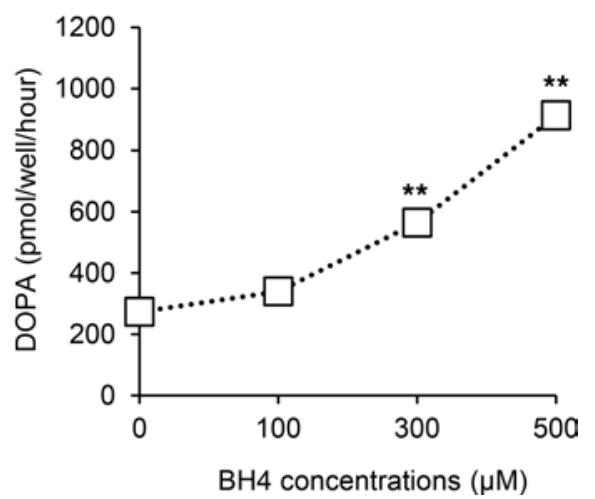

Figure 7. BH4 deficiency impairs noradrenaline synthesis and supplementation of BH4 increases noradrenaline syntheses. (A and B) Noradrenaline (NA) contents in brown adipose tissue (BAT) $(A ; n=6)$ and urine collected for 48 hours using individual metabolic cages (B; $n=$ 6-8) of hph-1 mice and control mice of the same background at 6 weeks of age. (C) Dihydroxyphenylalanine (DOPA) production in 120 minutes with PC12 cells after incubation with GTPCH I inhibitor (DAHP) for 16 hours $(n=3)$. (D) DOPA production in PC12 cells in the last 120 minutes in the presence of extracellular tetrahydrobiopterin $(\mathrm{BH} 4)$ for the total 240 minutes ( $n$ $=5$ ). Values are mean \pm SEM. Statistical analysis was performed by Student's $t$ test (A and $\mathbf{B}$ ). ${ }^{*} P<0.05$ vs. control group. Statistical analysis was also performed by 1-way ANOVA with Tukey post-hoc test (C and $\mathbf{D}) .{ }^{*} P<0.01$ vs. values without DAHP or $\mathrm{BH} 4$.

mice are being used as an eNOS uncoupling model mouse $(31,50)$, and supplementation of $\mathrm{BH} 4$ to these mice has been shown to ameliorate eNOS dysfunction and to increase NO levels in BAT. Moreover, administration of MTX, which is an inhibitor of DHFR, an enzyme that converts $\mathrm{BH} 2$ to $\mathrm{BH} 4$, induces further reduction of the $\mathrm{BH} 4 / \mathrm{BH} 2$ ratio and further deterioration of BAT function and glucose metabolism. These results suggest that $\mathrm{NO}$ critically participates in the regulation of BAT function by BH4. We therefore investigated whether the effect of $\mathrm{BH} 4$ on BAT regulation disappeared in eNOS-deficient mice. However, $\mathrm{BH} 4$ also increased BAT function in eNOS-deficient mice to some extent. These data indicate the existence of factors other than eNOS that account for BH4-induced BAT function. As another factor, NA is implicated because NA stimulation via the $\beta_{3}$-adrenergic receptor is well known as a BAT activator $(10,23,24,51)$. We confirmed the reduction of NA levels in BAT and urine of hph-1 mice and the correlation between BH4 and DOPA levels in PC12 cells. These findings strongly indicate that BH4 regulates BAT via both NA and NO. From this study, however, it is difficult to clarify which factors play essential or complementary roles in terms of the regulation of BAT and energy metabolism; further investigation is required.

Regarding the relationship between $\mathrm{BH} 4$ and glucose metabolism, we previously reported that $\mathrm{BH} 4$ has an eNOS-dependent suppressing effect on hepatic gluconeogenesis and that $\mathrm{BH} 4$ ameliorates glucose intolerance and insulin resistance in $o b / o b$ mice (47). As the expression level of GTPCH I and the level of $\mathrm{BH} 4$ de novo synthesis are highest in liver among those of respective tissues (45), it is estimated that liver has a contribution on glucose metabolism by BH4. However, we found in the present study that BH4 exerts a suppressing effect on hepatic gluconeogenesis via BAT using BAT transplantation from BH4-sufficient mice into BH4-deficient mice. Furthermore, this transplantation also affected lipid accumulation in liver, glucose tolerance, and insulin sensitivity. These data document the importance of the finding that BAT also contributes to glucose metabolism via $\mathrm{BH} 4$, including hepatic glucose metabolism.

Moreover, we found a direct effect of $\mathrm{BH} 4$ on brown adipocytes by a mechanism that may be dependent on NO derived from eNOS, which is abundantly expressed in brown adipocytes $(25,42)$. Recently, Chouch ani et al. reported that mitochondrial redox signaling regulates thermogenic response and energy expenditure via UCP1 activation in BAT (52). The main ROS involved in mitochondrial redox signaling would seem 


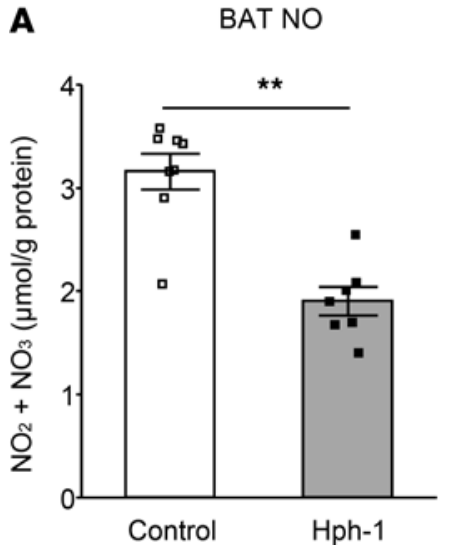

A

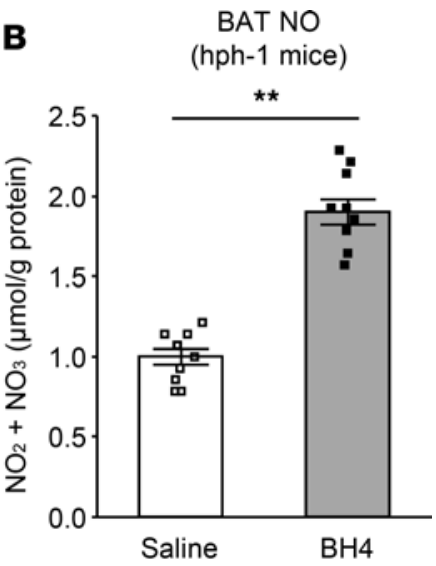

Figure 8. BH4 deficiency impairs NO synthesis and supplementation of BH4 increases NO syntheses. (A) NO contents of brown adipose tissue (BAT) in hph-1 mice and control mice of the same background at 6 weeks of age $(n=7-8)$. (B) NO contents of BAT in hph-1 mice after treatment with or without $20 \mathrm{mg}$ tetrahydrobiopterin $(\mathrm{BH} 4) / \mathrm{kg}$ body weight $(n=10)$. Values are mean \pm SEM. Statistical analysis was performed by Student's $t$ test. ${ }^{* *} P<0.01 \mathrm{vs}$. control or saline group.

to be $\mathrm{H}_{2} \mathrm{O}_{2}$; however, $\mathrm{NO}$ is also considered to contribute (53). Thus, it is possible that BH4-derived NO participates in redox modification-related regulation of BAT. It should be noted that we have not demonstrated how much the direct effect of $\mathrm{BH} 4$ on brown adipocytes contributes to the regulation of BAT in vivo. As we assessed the role of $\mathrm{BH} 4$ using global $\mathrm{BH} 4$-deficient mice in the present study, the limitations of the current study must be considered. Further investigations, e.g., by using BAT-specific GTPCH I-deficient model mice, are required to elucidate the BAT-specific effects of BH4 in the regulation of systemic energy and glucose metabolism.

As a cofactor of $\mathrm{PAH}, \mathrm{BH} 4$ is being used clinically as an orphan drug in patients with inherited $\mathrm{BH} 4$ deficiency and a particular type of phenylketonuria $(16,17)$. We show here the effects of $\mathrm{BH} 4$ on activation of BAT function in hph-1 mice using a dosage similar to that used in patients with phenylketonuria as a cofactor of PAH (17). NA induces increased BAT activity as well as some side effects, such as elevation of blood pressure and heart rate (51). On the other hand, over the last decade, $\mathrm{BH} 4$ research has been focused on improvement of endothelial dysfunction, and it is being used in clinical trials with patients with atherosclerotic diseases for the expected vasodilatation effects of $\mathrm{BH} 4$ through $\mathrm{NO}$ production (22). Although these effects indicate that $\mathrm{BH} 4$ has little effect on elevation of blood pressure or heart rate, as seen in some $\beta_{3}$-adrenergic receptor selective agonists (51), careful consideration is required in clinical use of $\mathrm{BH} 4$ for the treatment of diabetes and obesity. The present study proposes a concept of the relationship between BH4 and energy metabolism that suggests an approach to the prevention of the cardiovascular complications of obesity and diabetes induced by endothelial dysfunction as well as amelioration of the disease itself. In conclusion, BH4 regulates BAT function and systemic energy metabolism and has potential as a treatment for metabolic disorders such as obesity and diabetes.

\section{Methods}

Animals. Hph-1 and control mice (C57BL/ $6 \times \mathrm{CBA} / \mathrm{Ca})$ of the same background were a gift from Keith Hyland (Department of Neurochemistry, Institute of Metabolic Disease, Baylor University Medical Center, Dallas, Texas, USA). eNOS-deficient mice in the C57BL/6 mouse background were obtained from Jackson Laboratories. C57BL/6 (wild-type) mice were obtained from Shimizu.

All experiments were carried out with male mice maintained in a temperature-controlled $\left(25^{\circ} \mathrm{C} \pm 2^{\circ} \mathrm{C}\right)$ environment, with a 12-hour-light/dark cycle and free access to water and CFD (10\% fat, $20 \%$ protein, and $70 \%$ carbohydrate by energy) (catalog no. D12450B; Research Diets Inc.) or HFD (60\% fat, $20 \%$ protein, and 20\% carbohydrate by energy) (catalog no. D12492; Research Diets Inc.). At 4 weeks of age, hph-1 mice and control mice of the same background were fed CFD or HFD for 4 weeks, and body weight was measured once a week. After 4 weeks of CFD or HFD, blood glucose levels, liver TG, and total CHO (T-CHO) contents, and body temperature were measured. Core body temperature was measured by using a rectal thermometer (Bioresearch Inc.). Body fat imaging by CT scan and measurement of energy expenditure by indirect calorimetry were performed, as described previously (54). Fat volume was measured by collecting eWAT and iWAT from the left side of the body.

IPGTT. After CFD or HFD for 4 weeks, the mice were fasted overnight for 16 hours, and glucose $(1 \mathrm{~g} /$ $\mathrm{kg}$ ) was injected with $0.9 \%$ (wt/vol) sterile saline intraperitoneally. Blood glucose levels were measured at $0,30,60,90$, and 120 minutes after injection.

Insulin tolerance test. After CFD or HFD for 4 weeks, the mice were fasted 6 hours, and regular insulin (1 unit $/ \mathrm{kg}$ ) was injected with $0.9 \%$ (wt/vol) sterile saline intraperitoneally.

Pyruvate tolerance test. After CFD or HFD for 4 weeks, the mice were fasted for 16 hours, and pyruvate $(1 \mathrm{~g} / \mathrm{kg})$ was injected with $0.9 \%(\mathrm{wt} / \mathrm{vol})$ sterile saline intraperitoneally. 
A BAT-related gene expression

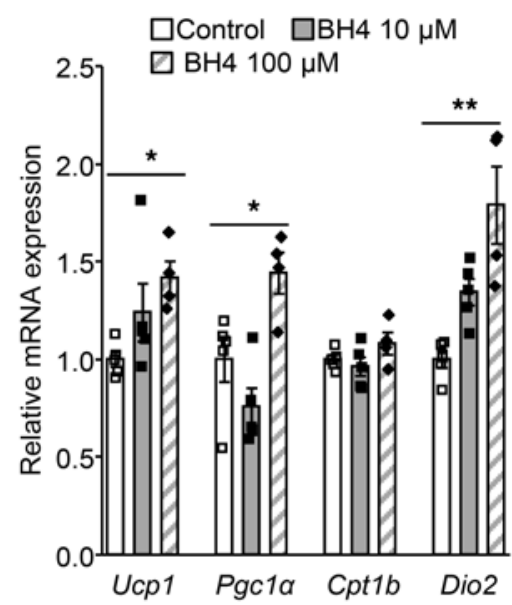

B UCP1 expression

UCP1

$\beta$-actin

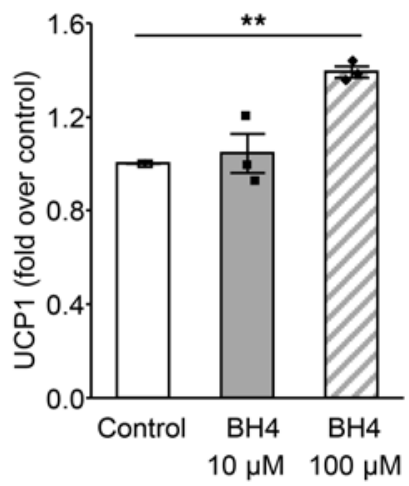

C

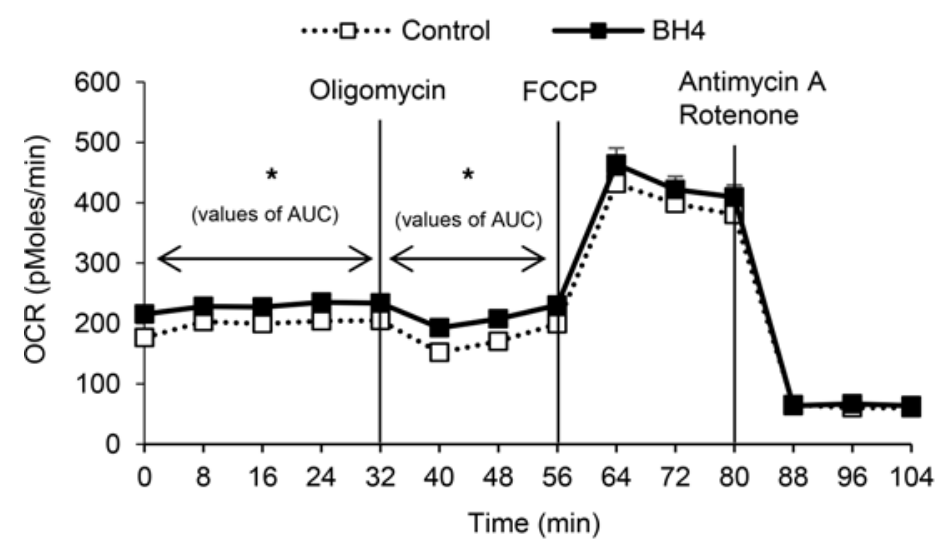

E

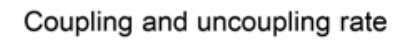

$\square$ Control $\square \mathrm{BH} 4$

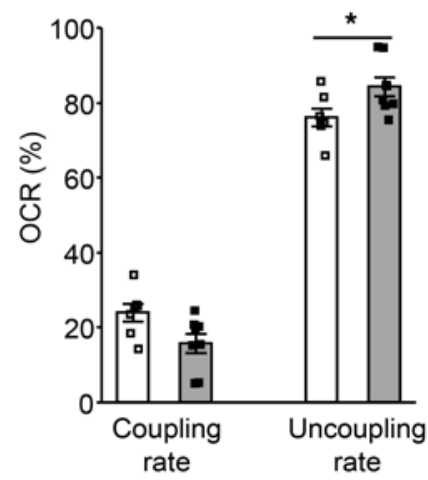

$\mathbf{F}$

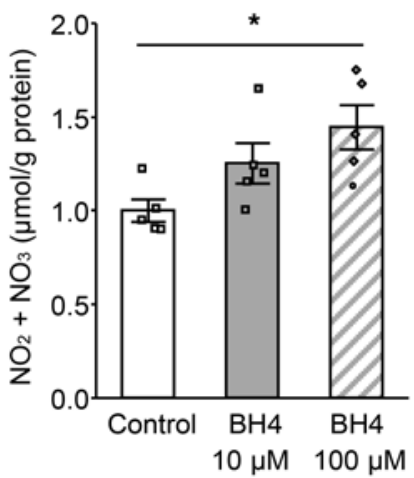

D $\begin{gathered}\text { Parameters of } \\ \text { mitochondrial function }\end{gathered}$

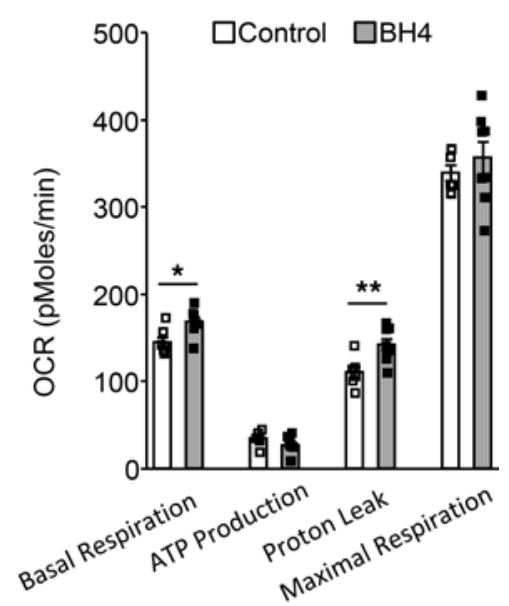

G

Ucp1 expression

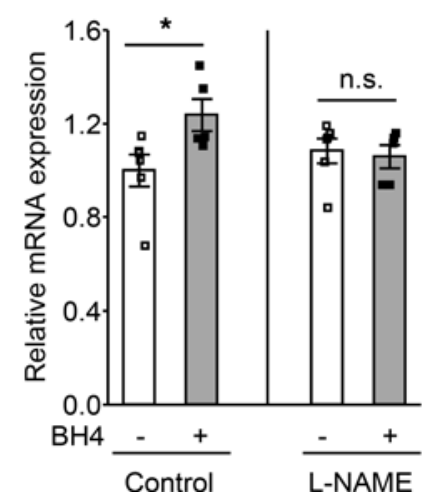

Figure 9. BH4 directly activates brown adipocytes NO-dependently. (A and B) mRNA levels of brown adipose tissue-related (BAT-related) genes by quantitative RT-PCR $(\mathbf{A} ; n=4-5)$ and protein expression levels of UCP1 $(\mathbf{B} ; n=3)$ in differentiated brown adipocytes on day 7 after incubated with or without 10 or $100 \mu \mathrm{M}$ tetrahydrobiopterin (BH4) for 24 hours. (C-E) Changes of oxygen consumption rate (OCR) (C); values of OCR related to basal respiration, ATP production, proton leak, and maximum respiration (D); and coupling and uncoupling rate (E) in the differentiated brown adipocytes on day 7 incubated with or without $100 \mu \mathrm{M} \mathrm{BH} 4$ for 24 hours $(n=7-8)$. (F) NO production in the differentiated brown adipocytes on day 7 incubated with or without 10 or $100 \mu \mathrm{M}$ BH4 for 24 hours $(n=5)$. (G) mRNA level of Ucp1 in the presence of the $100 \mu \mathrm{M}$ nitric oxide synthase (NOS) inhibitor (L-NAME) in the differentiated brown adipocytes on day 7 incubated with or without $100 \mu \mathrm{M} \mathrm{BH} 4$ for 24 hours $(n=5-6)$. Values are mean \pm SEM. Statistical analysis was performed by Student's $t$ test (C-E and $\mathbf{G}$ ). ${ }^{*} P<0.05,{ }^{* *} P<0.01$, NS vs. control group (C-E) or vs. values without BH4 (G). In addition, statistical analysis was performed by 2-way ANOVA (C) or 1-way ANOVA with Tukey post-hoc test (A, B, and F). ${ }^{*} P<0.05,{ }^{* *} P<0.01 \mathrm{vs}$. control group. 
CTT. Rectal temperature of mice was measured at $0,1,2.5,4$, and 5 hours after $4^{\circ} \mathrm{C}$ cold exposure. After an intraperitoneal single dose of BH4 $(20 \mathrm{mg} / \mathrm{kg}$ ) with $0.9 \%$ (wt/vol) sterile saline or $0.9 \%$ (wt/vol) sterile saline alone, rectal temperature was measured at $0,30,60$, and 90 minutes after $4^{\circ} \mathrm{C}$ cold exposure at 6 weeks of age in eNOS-deficient mice and hph-1 mice.

Morphological evaluation. Mouse interscapular BAT and eWAT samples were removed from hph-1 mice and control mice. An aorta sample was removed from these mice 4 weeks after the single injection of 120 $\mathrm{mg}$ streptozotocin $/ \mathrm{kg}$ body weight, as described previously (47). Isolated tissue samples were fixed in $10 \%$ neutral buffered formalin before processing through paraffin. Rehydrated paraffin sections were stained in hematoxylin solution and eosin solution. In isolated aorta samples, rehydrated paraffin sections were incubated overnight at $4^{\circ} \mathrm{C}$ with primary mouse anti-nitrotyrosine antibody (1:3,000 dilution; Enzo Life Sciences). Secondary antibodies used were goat anti-rabbit IgG (Life Technologies Japan). Images were taken using optical microscopy with FSX100 and FSX-BSW software (Olympus Life Science). White adipose cell size was assessed by measuring the diameter, and adiposity was calculated using FSX-BSW software.

To assess mitochondrial morphology, BAT samples of hph-1 mice and control mice were fixed in $4 \%$ formaldehyde and $2 \%$ glutaraldehyde in $0.1 \mathrm{M}$ phosphate buffer. These samples were cut into 1-mm cubes, and osmium fixation was performed. Images were taken using a transmission electron microscope (Hitachi Ltd.).

Quantitative RT-PCR. Total RNA was prepared by QIAzol Lysis Reagent (Qiagen), as described previously with slight modifications (55). The mouse sequences of forward and reverse primers to detect Gch1, Ucp1, PRD1-BF1-RIZ1 homologous domain-containing 16 (Prdm16), Pgc1 $\alpha$, Dio2, carnitine palmitoyl transferase $1 b(C p t 1 b)$, and acidic ribosomal phosphoprotein P0 (36B4) as an inner control are shown in Supplemental Table 1. SYBR Green PCR Master Mix (Applied Biosystems) was prepared for the quantitative RT-PCR run. The thermal cycling conditions were denaturation at $95^{\circ} \mathrm{C}$ for 10 minutes followed by 40 cycles at $95^{\circ} \mathrm{C}$ for 15 seconds and $60^{\circ} \mathrm{C}$ for 1 minute. mRNA levels were measured by real-time quantitative RT-PCR using ABI PRISM 7000 Sequence Detection System (Applied Biosystems).

Immunoblot analysis. Immunoblotting was performed as described previously with slight modifications (47). In short, isolated interscapular BAT, liver, and brown adipocytes were homogenized in lysis buffer. Cell lysates were heated at $95^{\circ} \mathrm{C}$ for 5 minutes and subjected to electrophoresis on $10 \%$ (vol/vol) sodium dodecyl sulfate-polyacrylamide gels and transferred onto nitrocellulose membranes. Primary antibodies used were anti-UCP1 (1:2,000 dilution; catalog U6382, Sigma-Aldrich), anti- $\beta$-actin (1:1,000 dilution; catalog 4967, Cell Signaling Technology), and anti-GTPCH I antibody (1:200 dilution; catalog sc-48510, Santa Cruz Biotechnology Inc.). The fluorescent bands were visualized using a detection system and quantified by densitometry (Amersham ECL Prime; GE Healthcare).

Effect of supplementation of BH4 in hph-1 mice. BH4 was obtained from Schircks Laboratories. At 5 weeks of age, blood glucose levels and body weight of hph-1 mice were measured, and the mice were divided into two groups, as shown in Supplemental Table 2. Each group was administered 0.9\% (wt/vol) sterile saline with or without BH4 (10 mg/kg) (Schircks Laboratories), which was freshly prepared and immediately injected intraperitoneally twice a day for 10 days. IPGTT ( $2 \mathrm{~g} / \mathrm{kg}$ glucose), measurement of body temperature, adipose cell size, and gene expressions in mRNA of BAT were performed.

BAT transplantation. BAT transplantation was performed by using hph-1 mice and control mice of the same background at 8 weeks of age. Donor BAT was isolated from hph-1 mice (BH4-deficient mice) and control mice (BH4-sufficient mice) and transplanted into hph-1 mice as recipient mice. In hph-1 mice recipient mice, rectal temperature, body weight, and fed blood glucose levels were measured, and the mice were divided into two groups transplanted with BAT derived from hph-1 mice or control mice. After cervical dislocation, $0.1 \mathrm{~g}$ BAT from the donor mice was removed from the intrascapular region, and the volume of the isolated BAT was measured and then incubated with saline at $37^{\circ} \mathrm{C}$ for 10 minutes. Under anesthetic by intraperitoneal injection of sodium pentobarbital, recipient mice were abdominally dissected on a $37^{\circ} \mathrm{C}$ heating plate, and BAT was transplanted into the visceral fat region (bilateral eWAT). After confirming the fixed position of eWAT, the BAT sample was deeply lodged and carefully wrapped with eWAT of the recipient mice. In this experiment, $0.05 \mathrm{~g}$ BAT was transplanted on each side of the eWAT, and a total of $0.1 \mathrm{~g}$ BAT was transplanted into the recipient mice. After BAT transplantation, recipient mice were housed with access to HFD for 20 days. Rectal temperature, body weight, and fed blood glucose were monitored every 10 days. CTT and measurement of fat volume, adipose cell size, liver TG and T-CHO, IPGTT ( $1 \mathrm{~g} / \mathrm{kg}$ glucose), insulin tolerance test ( 1 unit/ $\mathrm{kg}$ insulin), and pyruvate tolerance test $(1 \mathrm{~g} / \mathrm{kg})$ were performed. 
Mouse brown adipocytes. Mouse brown preadipocytes were isolated from mice with a C57BL/6 genetic background and were immortalized by SV40 large T antigen gene transfer. These mouse brown preadipocytes were maintained in DMEM containing $10 \% \mathrm{FBS}, 100 \mathrm{U} / \mathrm{ml}$ penicillin, and $100 \mu \mathrm{g} / \mathrm{ml}$ streptomycin at $37^{\circ} \mathrm{C}$ in $5 \% \mathrm{CO}_{2}$. The cells were incubated to differentiate by $10 \mu \mathrm{g} / \mathrm{ml}$ insulin, $0.5 \mu \mathrm{M}$ dexamethasone, $0.5 \mathrm{mM}$ 1-methyl-3-isobutylxanthine, $1 \mathrm{nM} \mathrm{T3}, 125 \mu \mathrm{M}$ indomethacin, and $0.5 \mu \mathrm{M}$ rosiglitazone (day 0 ). After 2 days, the differentiation medium was changed to the maintenance medium containing $5 \mu \mathrm{g} / \mathrm{ml}$ insulin and $1 \mathrm{nM}$ T3. The maintenance medium was changed every 2 days (days 2-6). At day 7, the adipocytes were treated with or without 10 or $100 \mu \mathrm{M} \mathrm{BH} 4$ and $100 \mu \mathrm{M}$ L-NAME (Sigma-Aldrich) for 24 hours (day 7).

Oxygen consumption rate in mouse brown adipocytes. Oxygen consumption rate (OCR), which indicates the mitochondrial respiration rate, was determined using an XF24 Extracellular Flux Analyzer (Seahorse Bioscience) (56), according to the manufacturer's instructions. Briefly, mouse brown adipocytes were seeded in 24-well plates (Seahorse Bioscience) and treated with or without $100 \mu \mathrm{M} \mathrm{BH} 4$ for 24 hours. The OCRs were calculated and recorded by a sensor cartridge and Seahorse XF-24 software. Following measurement of basal respiration, $500 \mathrm{nM}$ oligomycin, an inhibitor of mitochondrial ATP-synthase, $1 \mu \mathrm{M}$ carbonyl cyanide p-trifluoromethoxyphenylhydrazone (FCCP), an uncoupler of potent mitochondrial oxidative phosphorylation, and a mixture of $15 \mu \mathrm{M}$ antimycin $\mathrm{A}$, an inhibitor of the mitochondrial complex III, and $15 \mu \mathrm{M}$ rotenone, an inhibitor of the mitochondrial complex I, were injected sequentially. The OCR values related to basal respiration, ATP production, proton leak, maximum respiration, and coupling and uncoupling rate were calculated with the following formula: (basal respiration $=$ last point before injection of oligomycin - minimum value after antimycin $\mathrm{A}$ and rotenone, ATP production = last point before injection of oligomycin - minimum value after oligomycin, proton leak $=$ minimum value after oligomycin - minimum value after antimycin $\mathrm{A}$ and rotenone, maximum respiration $=$ maximum value after $\mathrm{FCCP}-$ minimum value after antimycin $\mathrm{A}$ and rotenone). Coupling rate and uncoupling rate were calculated with the following formula = (coupling rate $[\%]=$ ATP production/basal respiration, uncoupling rate $[\%]=100-$ coupling rate) .

Nitrite/nitrate analysis. Mouse BAT was isolated from hph-1 mice and control mice at 6 weeks of age. Sixty minutes after the intraperitoneal single dose of BH4 $(20 \mathrm{mg} / \mathrm{kg}$ ) with $0.9 \%$ (wt/vol) sterile saline or $0.9 \%$ (wt/vol) sterile saline alone, BAT was isolated from hph-1 mice, eNOS-deficient mice, and WT mice at 6 weeks of age. Mouse BAT and brown adipocytes treated with or without BH4 were homogenized in lysis buffer. Concentrations of nitrite and nitrate in the supernatant were evaluated by a fluorescence method (Dojindo).

$N A$ analysis. Mouse BAT was removed from hph-1 mice and control mice at 6 weeks of age. After measurement of tissue volume, isolated tissues were dipped into $1 \mathrm{ml} 0.4 \mathrm{M}$ perchloric acid, frozen in liquid nitrogen, and stored at $-80^{\circ} \mathrm{C}$. Dihydroxybenzylamine was added as an internal standard, and the tissue samples were homogenized. After centrifugation, the catecholamines in the supernatant were purified with activated alumina, as described previously (13). Urine samples were collected for 48 hours using individual metabolic cages. $1 \mathrm{ml} 6 \mathrm{M} \mathrm{HCl}$ solution was added to the urine sample and stored at $-20^{\circ} \mathrm{C}$. The urine samples were purified via activated alumina, as previously described (13).

Measurement of catecholamine synthesis rate in PC12 cells. PC12 cells (JCRB0733) obtained from the JCRB Cell Bank were maintained in DMEM containing 7\% each of FBS and horse serum. The cells were seeded at 200,000 cells/well of a 96-well plate (Corning 353072) 2 days before experiments. PC12 cells were pretreated with 0 or $0.1-10 \mathrm{mM}$ DAHP for 16 hours or with 0 and $100-500 \mu \mathrm{M} \mathrm{BH} 4$ for 120 minutes. The cells were then subjected to a metabolic arrest experiment in the presence of respective concentrations of DAHP or BH4. The metabolic arrest using $400 \mu \mathrm{M}$ NSD1015 (3-hydroxybenzylhydrazine dihydrochloride, Nacalai Tesque), an inhibitor of aromatic-L-amino acid decarboxylase, was performed as described previously (57), except that the incubation time was 120 minutes in the presence of NSD and DOPA determination followed instead of 5HTP measurement. DOPA was measured by HPLC-ECD equipped with Eicom SC-5ODS column. This method supplied us actual $\mathrm{TH}$ activity achieved under the conditions of the living cell interior, including respective substrate concentrations available, such as tyrosine and $\mathrm{BH} 4$.

Biopterin analysis. Tissues, whole blood, and urine were collected from hph-1 mice and control mice at 6 weeks of age. Under an anesthetic, the mice were abdominally dissected, and blood, urine, and liver and spleen tissues were collected. The organs were weighed, frozen immediately in liquid nitrogen, and stored at $-80^{\circ} \mathrm{C}$. Total biopterin, $\mathrm{BH} 4$, and the $\mathrm{BH} 4 / \mathrm{BH} 2$ ratio were measured as described previously $(47,58)$. 
Effect of administration of MTX in hph-1 mice. MTX, a DHFR inhibitor that inhibits BH4 biosyntheses in the salvage pathway (31), was obtained from Sigma-Aldrich. Blood glucose levels and body weight were measured in hph-1 mice at 5 weeks of age, and the mice were divided into two groups. After grouping, PBS with or without MTX (2 mg/kg) was injected intraperitoneally every 2 days for 2 weeks. BH4/BH2 ratio, rectal temperature, and fed blood glucose were measured.

Statistics. Comparison between two groups was performed using 2-tailed Student's $t$ test. One-way ANOVA with Tukey post-hoc test was performed when comparing more than two groups. Two-way ANOVA with Bonferroni post-hoc test was performed when comparing groups with repeated measures. Relationships between two groups were assessed using Spearman's rank correlation coefficient. $P<0.05$ was considered statistically significant.

Study approval. All experiments involving animals were conducted in accordance with the Guidelines for Animal Experiments of Kyoto University and were approved by the Animal Research Committee, Graduate School of Medicine, Kyoto University.

\section{Author contributions}

YO researched data, performed data analysis, contributed to discussion, and wrote, reviewed, and edited the manuscript. YF performed data analysis, contributed to discussion, and wrote, reviewed, and edited the manuscript. AA and A. Ohashi researched data, performed data analysis, and contributed to discussion. TG performed data analysis and contributed to discussion. FF, A. Obara, TF, NM, and MK performed data analysis. $\mathrm{MH}$ and TK contributed to discussion. HH contributed to discussion and reviewed the manuscript. NI contributed to discussion and wrote, reviewed, and edited the manuscript. NI is the guarantor of this work, had full access to all the data, and takes full responsibility for the integrity of the data and the accuracy of data analysis.

\section{Acknowledgments}

We wish to thank Keith Hyland of the Department of Neurochemistry, Institute of Metabolic Disease, Baylor University Medical Center, for the gift of hph-1 mice and control mice of the same background. We thank Tatsuaki Tsuruyama for technical assistance with morphological evaluation, and we are grateful for the use of a transmission electron microscope from the Center for Anatomical, Pathological and Forensic Medical Researches at Kyoto University. We thank Satoshi Morita of Department of Biomedical Statistics and Bioinformatics, Kyoto University Graduate School of Medicine, for assistance with statistical analysis. This study was supported by scientific research grants, Japan Society for the Promotion of Science (JSPS) KAKENHI (JP16J09973 and JP15K19514), a grant for Leading Project for Biosimulation from the Ministry of Education, Culture, Sports, Science, and Technology of Japan, a grant from the Japan Foundation for Applied Enzymology, and a grant from MSD Life Science Foundation.

Address correspondence to: Nobuya Inagaki, Department of Diabetes, Endocrinology and Nutrition, Graduate School of Medicine, Kyoto University, 54 Shogoin, Kawahara-cho, Sakyo-ku, Kyoto 606-8507, Japan. Phone: 81.075.751.3562; E-mail: inagaki@kuhp.kyoto-u.ac.jp.

1. Kahn BB, Flier JS. Obesity and insulin resistance. J Clin Invest. 2000;106(4):473-481.

2. $\mathrm{Ng} \mathrm{M}$, et al. Global, regional, and national prevalence of overweight and obesity in children and adults during 1980-2013: a systematic analysis for the Global Burden of Disease Study 2013. Lancet. 2014;384(9945):766-781.

3. Spiegelman BM, Flier JS. Obesity and the regulation of energy balance. Cell. 2001;104(4):531-543.

4. Tuomilehto J, et al. Prevention of type 2 diabetes mellitus by changes in lifestyle among subjects with impaired glucose tolerance. N Engl J Med. 2001;344(18):1343-1350.

5. Puzziferri N, Roshek TB, Mayo HG, Gallagher R, Belle SH, Livingston EH. Long-term follow-up after bariatric surgery: a systematic review. JAMA. 2014;312(9):934-942.

6. Saito M, et al. High incidence of metabolically active brown adipose tissue in healthy adult humans: effects of cold exposure and adiposity. Diabetes. 2009;58(7):1526-1531.

7. van Marken Lichtenbelt WD, et al. Cold-activated brown adipose tissue in healthy men. NEngl J Med. 2009;360(15):1500-1508.

8. Cypess AM, et al. Identification and importance of brown adipose tissue in adult humans. N Engl J Med. 2009;360(15):1509-1517.

9. Virtanen KA, et al. Functional brown adipose tissue in healthy adults. $N$ Engl J Med. 2009;360(15):1518-1525.

10. Kajimura S, Saito M. A new era in brown adipose tissue biology: molecular control of brown fat development and energy homeostasis. Annu Rev Physiol. 2014;76:225-249. 
11. Yoneshiro T, et al. Recruited brown adipose tissue as an antiobesity agent in humans. J Clin Invest. 2013;123(8):3404-3408

12. Hanssen MJ, et al. Short-term cold acclimation improves insulin sensitivity in patients with type 2 diabetes mellitus. Nat Med. 2015;21(8):863-865

13. Kim M, et al. Fish oil intake induces UCP1 upregulation in brown and white adipose tissue via the sympathetic nervous system. Sci Rep. 2015;5:18013.

14. Werner ER, Blau N, Thöny B. Tetrahydrobiopterin: biochemistry and pathophysiology. Biochem J. 2011;438(3):397-414.

15. Gross SS, Jaffe EA, Levi R, Kilbourn RG. Cytokine-activated endothelial cells express an isotype of nitric oxide synthase which is tetrahydrobiopterin-dependent, calmodulin-independent and inhibited by arginine analogs with a rank-order of potency characteristic of activated macrophages. Biochem Biophys Res Commun. 1991;178(3):823-829.

16. Curtius HC, et al. Atypical phenylketonuria due to tetrahydrobiopterin deficiency. Diagnosis and treatment with tetrahydrobiop terin, dihydrobiopterin and sepiapterin. Clin Chim Acta. 1979;93(2):251-262.

17. Blau N. Sapropterin dihydrochloride for the treatment of hyperphenylalaninemias. Expert Opin Drug Metab Toxicol. 2013;9(9):1207-1218.

18. Ichinose $\mathrm{H}$, et al. Hereditary progressive dystonia with marked diurnal fluctuation caused by mutations in the GTP cyclohydrolase I gene. Nat Genet. 1994;8(3):236-242.

19. Hyland K, Gunasekara RS, Munk-Martin TL, Arnold LA, Engle T. The hph-1 mouse: a model for dominantly inherited GTP-cyclohydrolase deficiency. Ann Neurol. 2003;54 Suppl 6:S46-S48.

20. Crabtree MJ, Channon KM. Synthesis and recycling of tetrahydrobiopterin in endothelial function and vascular disease. Nitric Oxide. 2011;25(2):81-88.

21. Vásquez-Vivar J, et al. Superoxide generation by endothelial nitric oxide synthase: the influence of cofactors. Proc Natl Acad Sci USA. 1998;95(16):9220-9225.

22. Katusic ZS, d'Uscio LV, Nath KA. Vascular protection by tetrahydrobiopterin: progress and therapeutic prospects. Trends Pharmacol Sci. 2009;30(1):48-54.

23. Lowell BB, Spiegelman BM. Towards a molecular understanding of adaptive thermogenesis. Nature. 2000;404(6778):652-660.

24. Bachman ES, et al. betaAR signaling required for diet-induced thermogenesis and obesity resistance. Science. 2002;297(5582):843-845.

25. Nisoli E, et al. Mitochondrial biogenesis in mammals: the role of endogenous nitric oxide. Science. 2003;299(5608):896-899.

26. Bode VC, McDonald JD, Guenet JL, Simon D. hph-1: a mouse mutant with hereditary hyperphenylalaninemia induced by ethylnitrosourea mutagenesis. Genetics. 1988;118(2):299-305.

27. Rothwell NJ, Stock MJ. A role for brown adipose tissue in diet-induced thermogenesis. Nature. 1979;281(5726):31-35.

28. Stanford KI, et al. Brown adipose tissue regulates glucose homeostasis and insulin sensitivity. J Clin Invest. 2013;123(1):215-223.

29. Nakanishi N, Onozawa S, Matsumoto R, Hasegawa H, Yamada S. Cyclic AMP-dependent modulation of vesicular monoamine transport in pheochromocytoma cells. J Neurochem. 1995;64(2):600-607.

30. Kietadisorn R, Juni RP, Moens AL. Tackling endothelial dysfunction by modulating NOS uncoupling: new insights into its pathogenesis and therapeutic possibilities. Am J Physiol Endocrinol Metab. 2012;302(5):E481-E495.

31. Crabtree MJ, Hale AB, Channon KM. Dihydrofolate reductase protects endothelial nitric oxide synthase from uncoupling in tetrahydrobiopterin deficiency. Free Radic Biol Med. 2011;50(11):1639-1646.

32. Förstermann U, Sessa WC. Nitric oxide synthases: regulation and function. Eur Heart J. 2012;33(7):829-837.

33. Liu X, et al. Brown adipose tissue transplantation improves whole-body energy metabolism. Cell Res. 2013;23(6):851-854.

34. NAGATSU T, LEVITT M, UDENFRIEND S. TYROSINE HYDROXYLASE. THE INITIAL STEP IN NOREPINEPHRINE BIOSYNTHESIS. J Biol Chem. 1964;239:2910-2917.

35. Kapatos G, Hirayama K, Hasegawa H. Tetrahydrobiopterin turnover in cultured rat sympathetic neurons: developmental profile, pharmacologic sensitivity, and relationship to norepinephrine synthesis. J Neurochem. 1992;59(6):2048-2055.

36. Cannon B, Nedergaard J. Brown adipose tissue: function and physiological significance. Physiol Rev. 2004;84(1):277-359.

37. Himms-Hagen J, et al. Effect of CL-316,243, a thermogenic beta 3-agonist, on energy balance and brown and white adipose tissues in rats. Am J Physiol. 1994;266(4 Pt 2):R1371-R1382.

38. Cai S, Khoo J, Channon KM. Augmented BH4 by gene transfer restores nitric oxide synthase function in hyperglycemic human endothelial cells. Cardiovasc Res. 2005;65(4):823-831.

39. Huang PL. eNOS, metabolic syndrome and cardiovascular disease. Trends Endocrinol Metab. 2009;20(6):295-302.

40. Elias I, et al. Adipose tissue overexpression of vascular endothelial growth factor protects against diet-induced obesity and insulin resistance. Diabetes. 2012;61(7):1801-1813.

41. Haas B, et al. Protein kinase G controls brown fat cell differentiation and mitochondrial biogenesis. Sci Signal. 2009;2(99):ra78

42. Giordano A, et al. Evidence for a functional nitric oxide synthase system in brown adipocyte nucleus. FEBS Lett. 2002;514(2-3):135-140.

43. Liu X, et al. Brown adipose tissue transplantation reverses obesity in ob/ob mice. Endocrinology. 2015;156(7):2461-2469.

44. Gunawardana SC, Piston DW. Reversal of type 1 diabetes in mice by brown adipose tissue transplant. Diabetes. 2012;61(3):674-682.

45. Hoshiga M, Hatakeyama K, Watanabe M, Shimada M, Kagamiyama H. Autoradiographic distribution of [14C]tetrahydrobiopterin and its developmental change in mice. J Pharmacol Exp Ther. 1993;267(2):971-978.

46. Stuehr D, Pou S, Rosen GM. Oxygen reduction by nitric-oxide synthases. J Biol Chem. 2001;276(18):14533-14536.

47. Abudukadier A, et al. Tetrahydrobiopterin has a glucose-lowering effect by suppressing hepatic gluconeogenesis in an endothelial nitric oxide synthase-dependent manner in diabetic mice. Diabetes. 2013;62(9):3033-3043.

48. Meininger CJ, et al. Impaired nitric oxide production in coronary endothelial cells of the spontaneously diabetic BB rat is due to tetrahydrobiopterin deficiency. Biochem J. 2000;349(Pt 1):353-356.

49. Gamez-Mendez AM, Vargas-Robles H, Ríos A, Escalante B. Oxidative stress-dependent coronary endothelial dysfunction in obese mice. PLoS One. 2015;10(9):e0138609.

50. Santhanam AV, d'Uscio LV, Smith LA, Katusic ZS. Uncoupling of eNOS causes superoxide anion production and impairs NO signaling in the cerebral microvessels of hph-1 mice. J Neurochem. 2012;122(6):1211-1218. 
51. Cypess AM, et al. Activation of human brown adipose tissue by a $\beta 3$-adrenergic receptor agonist. Cell Metab. 2015;21(1):33-38.

52. Chouchani ET, et al. Mitochondrial ROS regulate thermogenic energy expenditure and sulfenylation of UCP1. Nature. 2016;532(7597):112-116.

53. Moncada S, Erusalimsky JD. Does nitric oxide modulate mitochondrial energy generation and apoptosis? Nat Rev Mol Cell Biol. 2002;3(3):214-220.

54. Nasteska D, et al. Chronic reduction of GIP secretion alleviates obesity and insulin resistance under high-fat diet conditions. Diabetes. 2014;63(7):2332-2343.

55. Ogura M, et al. Overexpression of SIRT5 confirms its involvement in deacetylation and activation of carbamoyl phosphate synthetase 1. Biochem Biophys Res Commun. 2010;393(1):73-78.

56. Loft A, et al. Browning of human adipocytes requires KLF11 and reprogramming of PPAR $\gamma$ superenhancers. Genes Dev. 2015;29(1):7-22.

57. Hasegawa H, Oguro K, Naito Y, Ichiyama A. Iron dependence of tryptophan hydroxylase activity in RBL2H3 cells and its manipulation by chelators. Eur J Biochem. 1999;261(3):734-739.

58. Sawabe K, Wakasugi KO, Hasegawa H. Tetrahydrobiopterin uptake in supplemental administration: elevation of tissue tetrahydrobiopterin in mice following uptake of the exogenously oxidized product 7,8-dihydrobiopterin and subsequent reduction by an anti-folate-sensitive process. J Pharmacol Sci. 2004;96(2):124-133. 